\title{
Evolution of polycyclic aromatic hydrocarbons in photodissociation regions
}

\section{Hydrogenation and charge states}

\author{
J. Montillaud ${ }^{1,2,3}$, C. Joblin ${ }^{1,2}$, and D. Toublanc ${ }^{1,2}$ \\ ${ }^{1}$ Université de Toulouse, UPS-OMP, IRAP, 31400 Toulouse, France \\ e-mail: christine.joblin@irap.omp.eu \\ 2 CNRS, IRAP, 9 Av. Colonel Roche, BP 44346, 31028 Toulouse Cedex 4, France \\ 3 Department of Physics, PO Box 64, 00014 University of Helsinki, Finland
}

Received 16 November 2012 / Accepted 23 January 2013

\section{ABSTRACT}

\begin{abstract}
Context. Various studies have emphasised variations in the charge state and composition of the interstellar polycyclic aromatic hydrocarbon (PAH) population in photodissociation regions (PDRs). These changes are expected to affect the energetics and chemistry in these regions, thereby calling for a quantitative description.

Aims. We aim to model the spatial evolution of the charge and hydrogenation states of PAHs in PDRs. We focus on the specific case of the north-west (NW) PDR of NGC 7023, for which many observational constraints are available. We also discuss the case of the diffuse interstellar medium (ISM).

Methods. We modelled the physical conditions in NGC 7023 NW using a state-of-the-art PDR code. We then used a new PAH chemical evolution model that includes recent experimental data on PAHs and describes multiphoton events. We considered a family of compact PAHs bearing up to 96 carbon atoms.

Results. The calculated ionization ratio is in good agreement with the observed ratio in NGC 7023 NW. Within the PDR, PAHs evolve into three major populations. We find medium-sized PAHs $\left(50 \lesssim N_{\mathrm{C}} \lesssim 90\right)$ to be normally hydrogenated, while larger PAHs $\left(N_{\mathrm{C}} \gtrsim 90\right)$ can be superhydrogenated, and smaller species $\left(N_{\mathrm{C}} \lesssim 50\right)$ are fully dehydrogenated. In the more diffuse gas of the cavity, where the fullerene $\mathrm{C}_{60}$ has recently been detected, all the studied PAHs are found to be quickly fully dehydrogenated. PAH chemical evolution exhibits a complex non-linear behaviour as a function of the UV radiation field because of multiphoton events. Steady state for hydrogenation is reached on timescales ranging from less than a year for small PAHs, up to $10^{4}$ years for large PAHs at $A_{V}=1$. Critical reactions that would need more studies are the recombination of cations with electrons, the reactivity of cations with $\mathrm{H}_{2}$, and the reactivity of neutral PAHs with $\mathrm{H}$.

Conclusions. We have developed a new model of PAH chemical evolution based on the most recent available molecular data. This model allows us to rationalise the observational constraints without any fitting parameter. PAHs smaller than 50 carbon atoms are not expected to survive in the NGC 7023 NW PDR. A similar conclusion is obtained for the diffuse ISM. Carbon clusters turn out to be end products of $\mathrm{PAH}$ photodissociation, and the evolution of these clusters needs to be investigated further to evaluate their impact on the chemical and physical evolution of PDRs.
\end{abstract}

Key words. ISM: molecules - dust, extinction - photon-dominated region (PDR) - astrochemistry

\section{Introduction}

Polycyclic aromatic hydrocarbons (PAHs) are now widely accepted as the carriers of the ubiquitous aromatic infrared bands (AIBs) observed at 3.3, 6.2, 7.7, 8.6, and $11.3 \mu \mathrm{m}$, and this has motivated a lot of studies of the properties of these species (see Joblin \& Tielens 2011, for a recent compilation of papers on this subject). Interstellar PAHs can contain up to $20 \%$ of the cosmic carbon and are the smallest dust particles by size. As such, they play a major role in the physics and chemistry of photodissociation regions (PDR) through the absorption of UV light (Joblin et al. 1992) and the photoelectric heating (Bakes \& Tielens 1994), and may also be involved in the formation of $\mathrm{H}_{2}$ (Habart et al. 2003).

The energetics of PDRs is well understood in its main lines. However, it has been known for more than a decade now (see, e.g. Hollenbach \& Tielens 1999) that the excitation temperatures observed for some molecular tracers are higher than predicted by
PDR models. This issue has been raised again recently by several authors who compared the results of the most recent PDR modelling tools with observational data from the last generation of spatial facilities (Spitzer, Herschel). These authors report difficulties accounting for the observed emission in $\mathrm{H}_{2}$ and high- $J$ CO rotational lines (Habart et al. 2011; Goicoechea et al. 2011; Joblin et al., in prep.). Habart et al. (2011) conclude that this is due to our poor understanding of the energetics of PDRs. The main heating mechanism at the surface of PDRs comes from the thermalisation of hot electrons generated by the photoelectric effect on PAHs and very small grains. The photoelectric efficiency depends on the grain charge and is expected to decrease when PAHs are positively ionized (Hollenbach \& Tielens 1999). This theoretical prediction has been recently supported by an analysis of observations (Okada et al. 2013).

The question of the exact evolution of PAH properties with physical conditions has been addressed recently by a combination of observations and theoretical modelling. The spatial 
evolution of the mid-IR spectra was observed in several regions with the Infrared Space Observatory (ISO) and the Spitzer telescope. Rapacioli et al. (2005) and Berné et al. (2007) used global decomposition methods to analyse the data, and they propose an evolutionary scenario in which a population of very small grains evaporates under the action of UV photons, releasing neutral free PAHs, which are subsequently photoionized. In addition, the recent detection of fullerene $\mathrm{C}_{60}$ in reflection nebulae (Sellgren et al. 2010) may be interpreted as a new step in this evolutionary scenario (Berné \& Tielens 2012).

In parallel with these observational studies, several models have been developed to describe the chemical evolution of PAHs. Bakes \& Tielens (1994) focused on the charge evolution to compute the contribution of PAHs to the gas heating by the photoelectric effect. A more comprehensive model was proposed by Allain et al. (1996a), which includes the photodestruction of PAHs exposed to UV photons, and it provides insight into the stability of interstellar PAHs, as a function of their size. Le Page et al. (2001) propose a new model dedicated to the charge and hydrogenation states of PAHs in the diffuse interstellar medium (ISM). Based on these models, Visser et al. (2007) built a comprehensive model of PAH evolution in protoplanetary disks, which includes the charge and hydrogenation states. The authors also describe the photodestruction of the skeleton of PAHs including multiphoton events that are frequent in these high-UV irradiation conditions.

In this paper, we present a new model that is inspired by Le Page et al. (2001) and that includes multiphoton events for all photodissociation processes. This model takes advantage of the latest available molecular data from both theoretical calculations (Malloci et al. 2007, 2008) and experimental measurements (Biennier et al. 2006; Betts et al. 2006; Joblin et al., in prep.). We apply our model to the prototypical north-west PDR (hereafter NW PDR) of NGC 7023 in an attempt to (i) identify key processes in the evolution of the charge and hydrogenation states of interstellar PAHs in order to provide guidelines for fundamental studies and to (ii) provide a quantitative description of the evolution of the PAH population, which is necessary for modelling the role of PAHs in the physics and chemistry of PDRs. Furthermore, the obtained results could guide the spectroscopic identification of individual PAHs, for instance, by matching some of the diffuse interstellar bands (see, for example, Salama et al. 2011). This motivates additional calculations of the hydrogenation and charge states of PAHs using a grid of conditions that include typical conditions for the diffuse ISM.

This work is organised as follows. The studied PDR and its physical conditions are presented in Sect. 2. In Sect. 3, molecular processes relevant to this study are reviewed and discussed. Our numerical model is presented in Sect. 4 and its results in Sect. 5. The sensitivity of the results to both molecular data and astrophysical conditions are investigated in Sect. 6. Consequences of our results on the direct detection of individual PAH species and on the fate of carbon clusters are discussed in Sect. 7.

\section{Astrophysical environment}

Our study focusses on the reflection nebula NGC 7023 that has been widely studied at many wavelengths (e.g. Rogers et al. 1995; Fuente et al. 1996, 1999; Gerin et al. 1998; Berné et al. 2008; Joblin et al. 2010). The region is part of a small molecular cloud at $430 \mathrm{pc}$, illuminated by the Be star HD $200775\left(\operatorname{RA}(2000)=21 \mathrm{~h} 01 \mathrm{~m} 36.9 \mathrm{~s} ; \operatorname{Dec}(2000)=+68^{\circ} 0947.8\right)$. It has been shaped by the star formation process leading to
Table 1. Input parameters of the Meudon PDR code (Le Bourlot et al. 2012) used to model the physical and chemical structure of NGC 7023 NW PDR.

\begin{tabular}{lccc}
\hline \hline Parameters & & Values & Units \\
\hline Star surface temp. & $T_{\text {eff }}$ & 15000 & $\mathrm{~K}$ \\
UV radiation field & $G_{0}$ & 2600 & $\mathrm{Habing}^{\dagger}$ \\
Fixed pressure & $P$ & $7 \times 10^{6}$ & $\mathrm{~K} \mathrm{~cm}^{-3}$ \\
$A_{V} / E_{B-V}$ & $R_{V}$ & 5.56 & \\
\hline & $x_{0}$ & 4.60 & $\mu \mathrm{m}^{-1}$ \\
Extinction curve with & $\gamma$ & 1.36 & $\mu \mathrm{m}^{-1}$ \\
Fitzpatrick \& Massa & $c_{1}$ & 0.80 & \\
parameters & $c_{2}$ & 0.32 & $\mu \mathrm{m}^{-2}$ \\
for HD 200775 & $c_{3}$ & 3.09 & $\mu \mathrm{m}^{-2}$ \\
& $c_{4}$ & 0.37 & \\
\hline CR ionization rate & $\zeta$ & $5 \times 10^{-17}$ & $\mathrm{~s}^{-1}$ \\
Dust minimum radius & $a_{\min }$ & $3 \times 10^{-7}$ & $\mathrm{~cm}$ \\
Dust maximum radius & $a_{\max }$ & $3 \times 10^{-5}$ & $\mathrm{~cm}$ \\
MRN distribution index & $\alpha$ & 3.5 & \\
\hline
\end{tabular}

Notes. ${ }^{(\dagger)}$ The fluxes are normalised by the flux of the interstellar radiation field between 912 and $2400 \AA$ measured by Habing (1968) in the $\sim 1 \mathrm{kpc}$ neighbourhood of the Sun.

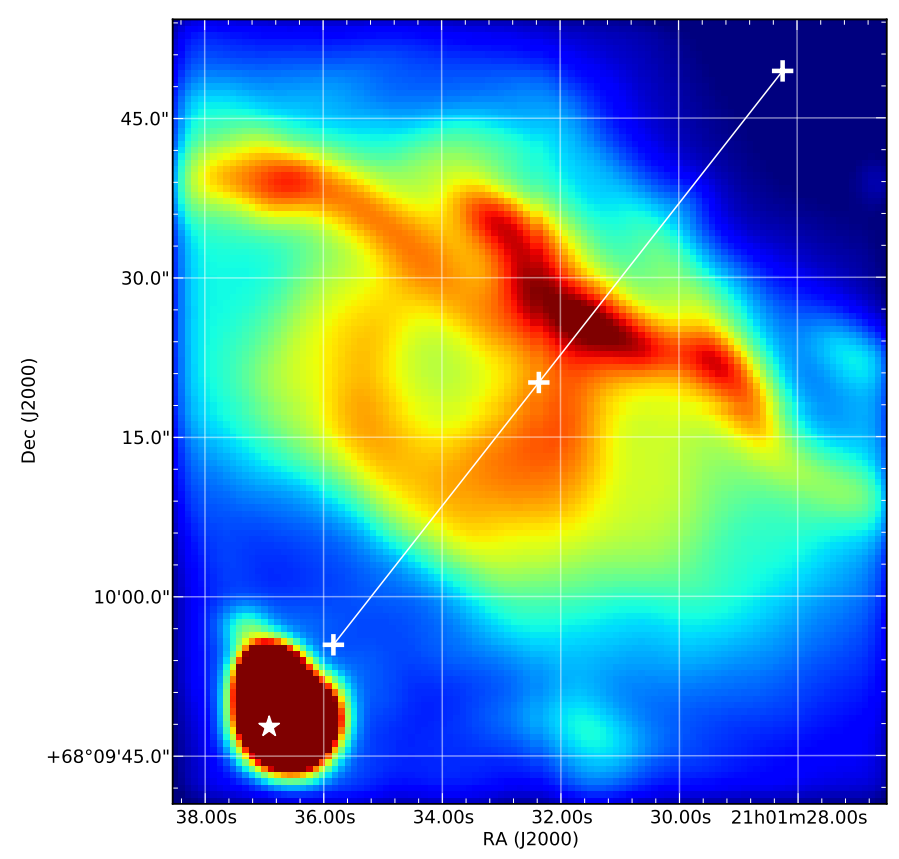

Fig. 1. Map of the NW PDR of NGC 7023 observed by SpitzerIRAC at $8 \mu \mathrm{m}$. The white star shows the location of the exciting star HD 200775. The white line shows the Star-NW cut that is studied in this paper, and the white crosses indicate the positions at 10, 42, and 80" from the star.

the formation of a cavity surrounded by denser filaments and clumps. Figure 1 shows the NW part of this region.

Pilleri et al. (2012) used their spectral fitting tool PAHTAT to analyse, in this region, the AIB emission that was recorded by the InfraRed Spectrograph (IRS) on-board Spitzer. They derived the respective contributions of $\mathrm{PAH}$ cations $\left(\mathrm{PAH}^{+}\right)$, neutral PAHs $\left(\mathrm{PAH}^{0}\right)$, as well as the so-called evaporating very small grains (eVSG). Figure 2 reports these values along the StarNW cut shown in Fig. 1. In the following, we focus on two regions of the nebula along the Star-NW cut: the cavity, where 
Table 2. Local physical conditions in the cavity of NGC 7023.

\begin{tabular}{ccccc}
\hline \hline $\begin{array}{c}d \\
{\left[{ }^{\prime \prime}\right]}\end{array}$ & $\begin{array}{c}T \\
{[\mathrm{~K}]}\end{array}$ & $\begin{array}{c}n(\mathrm{H}) \\
{\left[\mathrm{cm}^{-3}\right]}\end{array}$ & $\begin{array}{c}n\left(\mathrm{H}_{2}\right) \\
{\left[\mathrm{cm}^{-3}\right]}\end{array}$ & $\begin{array}{c}n\left(\mathrm{e}^{-}\right) \\
{\left[\mathrm{cm}^{-3}\right]}\end{array}$ \\
\hline 10 & 103 & 150 & $6.9 \times 10^{-8}$ & $3.7 \times 10^{-2}$ \\
20 & 129 & 150 & $6.8 \times 10^{-7}$ & $3.9 \times 10^{-2}$ \\
30 & 150 & 150 & $3.3 \times 10^{-6}$ & $4.0 \times 10^{-2}$ \\
\hline
\end{tabular}

the emission of fullerene $\mathrm{C}_{60}$ is detected and the AIB signal disappears (Sellgren et al. 2010; Berné \& Tielens 2012) and the well-studied NW PDR, where the AIB emission is intense.

To provide a realistic description of the spatial evolution of physical conditions (density and gas temperature) and chemical abundances in the PDR, we used the Meudon PDR code (version 1.4.3, rev. 608, Le Bourlot et al. 2012). The input parameters are summarised in Table 1 . The illuminating star was modelled using a synthetic stellar atmosphere spectrum from Castelli \& Kurucz (2004) with an effective temperature of $15000 \mathrm{~K}$ (solar elemental abundances and $\log (g)=4.0)$ and an integrated UV intensity of 2600 in units of Habing (Habing 1968) at 42" from the star (Joblin et al. 2010). The UV spectrum measured with IUE and the photometric $U, B, V$ (Mermilliod et al. 1997), and $J$ (Skrutskie et al. 2006) bands were combined with this stellar atmosphere to determine the Fitzpatrick and Massa parameters of the extinction curve along the line of sight toward HD 200775 (Fitzpatrick \& Massa 2005). These parameters are used for radiative transfer calculations in the PDR code.

For the NW PDR, we computed an isobaric model with a thermal pressure of $7 \times 10^{6} \mathrm{~K} \mathrm{~cm}^{-3}$, which provides a density profile in reasonable agreement with the profile derived by Pilleri et al. (2012) from the Spitzer mid-IR observations, and also with the $\mathrm{C}^{+}$emission at $158 \mu \mathrm{m}$ measured with Herschel/HIFI at this location (Joblin et al. 2010). For the cavity, we computed isochore models for three positions at 10, 20, and 30" from the star. We followed Berné \& Tielens (2012) and considered a density of hydrogen nuclei $n_{\mathrm{H}}=150 \mathrm{~cm}^{-3}$ and a local $\mathrm{UV}$ radiation field that is derived by applying a geometrical dilution factor.

The resulting physical conditions and chemical abundances are shown in Fig. 2 as a function of the position along the Star-NW cut for the NW PDR. Since the extinction is negligible in the cavity, the variations in the radiation field are dominated by the geometrical dilution that cannot be taken into account using the plane-parallel geometry of the Meudon PDR code. Therefore, for each position in the cavity, we ran a different model and checked that the extinction was negligible along distances of the order of $10^{\prime \prime}$. In the following, we use the local conditions obtained at $A_{V}=10^{-4}$ in each cavity model, as summarised in Table 2, and compute the evolution of PAHs in this region. In the next section, we present the physical and chemical processes that drive the evolution of PAH charge and hydrogenation states.

\section{Physical and chemical processes}

In the following, we focus on the evolution of the charge and hydrogenation states of a few PAHs, namely coronene $\left(N_{\mathrm{C}}=24\right)$, circumcoronene $\left(N_{\mathrm{C}}=54\right)$, circumovalene $\left(N_{\mathrm{C}}=66\right)$, and circumcircumcoronene $\left(N_{\mathrm{C}}=96\right)$. These molecules have compact geometries and are therefore expected to be the most stable for a given size. Furthermore, coronene is the largest PAH species whose chemical properties have been studied experimentally (Bierbaum et al. 2011).
We review here our current knowledge of the main processes that drive the evolution of these PAHs and justify our choices when facing the lack of available molecular data. We do not consider the gain or loss of $\mathrm{C}$-atoms in this section because (i) the reactivity with gas-phase species such as $\mathrm{C}^{+}$and $\mathrm{O}$ is expected to be much slower than the processes related to charge and hydrogenation states (Le Page et al. 2001), and (ii) large and compact PAHs were shown to primarily lose all their hydrogen atoms before enduring C-loss when submitted to photodissociation (Ekern et al. 1998).

In the following, we define the hydrogenation state as the number $N_{\mathrm{H}}$ of $\mathrm{H}$-atoms bound to the carbon skeleton of the molecule. We disentangle (i) normally hydrogenated PAHs, containing the maximum number $N_{\mathrm{H}}^{0}$ of $\mathrm{H}$-atoms bound to peripheral C-atoms without breaking aromaticity, (ii) dehydrogenated PAHs that have lost one or several $\mathrm{H}$-atoms compared to the normally hydrogenated structure $\left(N_{\mathrm{H}}<N_{\mathrm{H}}^{0}\right)$, and (iii) superhydrogenated PAHs with more $\mathrm{H}$-atoms than in the normally hydrogenated structure $\left(N_{\mathrm{H}}>N_{\mathrm{H}}^{0}\right)$, leading to some aliphatic bonds. In practice, we will only consider species with $N_{\mathrm{H}}=N_{\mathrm{H}}^{0}+1$ since there is no quantitative data on more hydrogenated species.

\subsection{Absorption of UV-visible photons}

The UV-visible absorption cross-sections of PAHs containing a few tens of $\mathrm{C}$-atoms have been investigated experimentally (Verstraete et al. 1990; Joblin et al. 1992; Ruiterkamp et al. 2002). Larger species are difficult to handle experimentally and their cross-sections are more easily obtained with theoretical methods, such as density functional theory (Malloci et al. 2004). One general result of these studies is that UV-visible absorption cross-sections scale with the number of carbon atoms in PAHs.

In this work, we use the cross-sections from the theoretical spectral database of PAHs (Malloci et al. 2007), because it provides us with a consistent set of data. Since there are no calculations for circumcircumcoronene $\left(\mathrm{C}_{96} \mathrm{H}_{24}\right)$, we scaled, according to the number of $\mathrm{C}$-atoms, the absorption cross-section of circumcoronene $\left(\mathrm{C}_{54} \mathrm{H}_{18}\right)$, the largest studied $\mathrm{PAH}$ with similar symmetry.

\subsection{Photoionization}

The photoionization cross-sections of pyrene $\left(\mathrm{C}_{16} \mathrm{H}_{10}\right)$ and coronene $\left(\mathrm{C}_{24} \mathrm{H}_{12}\right)$ were measured by Verstraete et al. (1990), who found that they are well parametrised by the number of $\mathrm{C}$-atoms and the ionization potential (IP). We made use of the formula proposed by Le Page et al. (2001):

$\sigma_{\text {ion }}=\sigma_{\mathrm{UV}} \times Y_{\text {ion }}=\sigma_{\mathrm{UV}} \times a \times \exp \left\{-b\left[c\left(E_{\mathrm{exc}}-d\right)\right]^{4}\right\}$

where the UV-visible absorption cross-section $\sigma_{\mathrm{UV}}$ is the one discussed in the previous section and the one that accounts for the evolution with the number of $\mathrm{C}$-atoms. The ionization yield $Y_{\text {ion }}$ accounts for the effect of the IP. The constants are taken from Le Page et al. (2001): $a=0.8, b=0.00128$, $c=\left(14.89-\mathrm{IP}_{\text {coronene }}\right) /\left(14.89-\mathrm{IP}_{\mathrm{PAH}}\right)$ where the ionization potential of coronene (IP $\left.\mathrm{I}_{\text {coronene }}\right)$ and of the studied PAH (IP $\left.\mathrm{PAH}_{\mathrm{PA}}\right)$ are expressed in $\mathrm{eV}$, and $d=14.89 \mathrm{eV}$. For coronene, circumcoronene, and circumovalene, we used the IP computed by Malloci et al. (2007) (7.02, 6.14, and $5.71 \mathrm{eV}$, respectively). For circumcircumcoronene, we assumed an IP of $5.68 \mathrm{eV}$, consistent with the parametrisation proposed by Ruiterkamp et al. (2005) in their Table B. 1 for molecules with a $D_{6 \mathrm{~h}}$ geometry. 

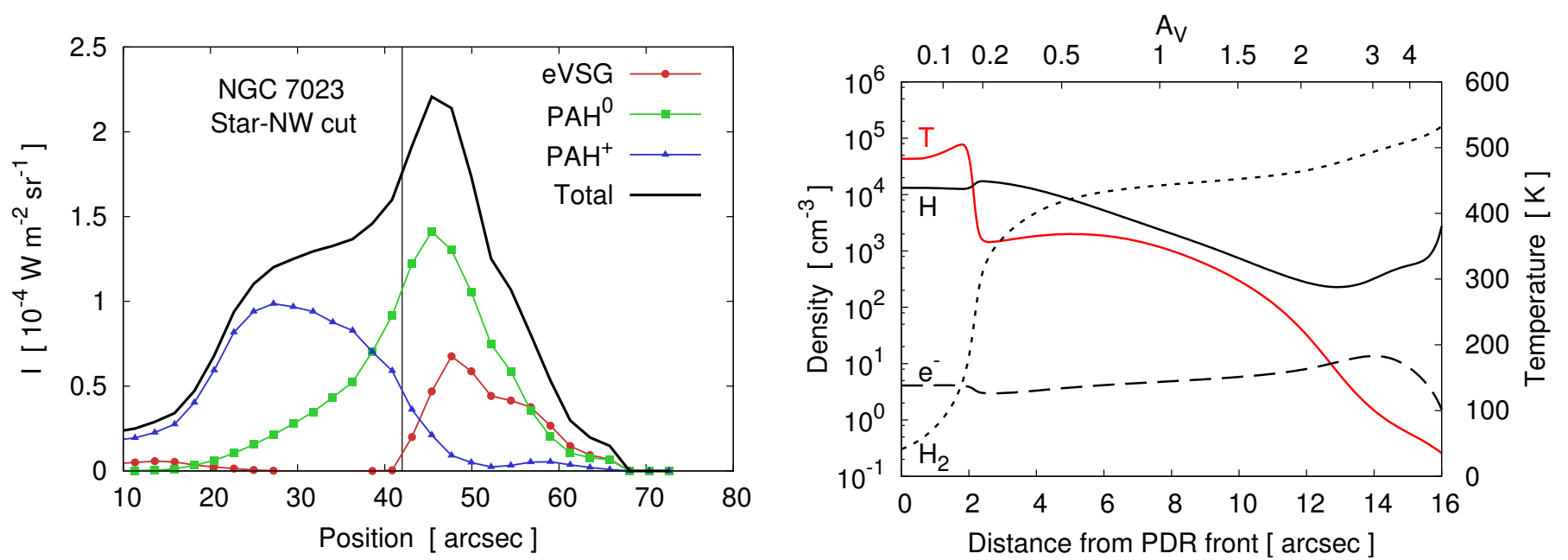

Fig. 2. Left: analysis of the AIB emission in NGC 7023 along the Star-NW cut, using the PAHTAT tool (Pilleri et al. 2012). The integrated intensity over the 5.5-14 $\mu \mathrm{m}$ spectral range after correction from extinction (thick black), as well as the contributions from eVSGs (red), neutral PAHs (green), and PAH cations (blue) are shown. The vertical line indicates the position of the PDR front, at $42^{\prime \prime}$ from the star. Right: physical and chemical structure of the NW PDR as computed with the Meudon PDR code, assuming an intensity of the UV radiation field $G_{0}=2600$ in Habing units at $A_{V}=0$ ( $42^{\prime \prime}$ from the star), and a constant pressure of $7 \times 10^{6} \mathrm{~K} \mathrm{~cm}^{-3}$.

\subsection{Photodissociation}

As discussed by Allain et al. (1996b), after the absorption of a UV-visible photon, a PAH can undergo several transformations involving a wealth of processes. It can lose an electron or a fragment $\left(\mathrm{H}, \mathrm{H}_{2}, \mathrm{C}_{2} \mathrm{H}_{2}\right.$, etc.), or relax energy by radiating IR photons or by luminescence. Allain et al. (1996b) showed that the branching ratios between the different pathways vary with the internal energy and the size of PAHs. For compact PAHs as studied here, the main processes, apart from photoionization, are $\mathrm{H}$-loss and IR photon emission. In the following, we therefore describe the photophysics of PAHs with a few simplified steps: (i) absorption of a UV-visible photon of energy $h v$ either leads to ionization with the branching ratio $Y_{\text {ion }}(h v)$ or increases, by the energy $h v$, the internal energy of the PAH, which is then found in a vibrationally excited state of the electronic ground state; (ii) emission of IR photons at the rate $k_{\mathrm{IR}}(E)$, with $E$ the internal energy of the PAH; (iii) fragmentation by $\mathrm{H}$-atom lost at the rate $k_{\text {diss }}(E)$. In this section, we present how we determined these two rates.

\subsubsection{IR relaxation}

Several approaches have been proposed to compute the IR photon emission rate of PAHs, from the simple assumption that $k_{\mathrm{IR}} \approx 10^{2} \mathrm{~s}^{-1}$ (Herbst \& McEwan 1990), up to a detailed Monte-Carlo kinetics model using a microcanonical formalism by Joblin et al. (2002). The latter approach requires the full knowledge of the vibrational mode frequencies and associated Einstein coefficients. The values of $k_{\mathrm{IR}}(E)$ are obtained by averaging typically over 10000 trajectories. For coronene, circumcoronene and circumovalene, we used this method and the vibrational properties from the theoretical spectral database of PAHs (Malloci et al. 2007). As can be seen in Fig. 3, the dependence of $k_{\mathrm{IR}}$ on PAH size is not strong. For circumcircumcoronene, we used the same values of $k_{\mathrm{IR}}$ as for circumcoronene.

In addition to the determination of $k_{\mathrm{IR}}(E)$, we extended the method of Joblin et al. (2002) to derive the mean energy $e_{\mathrm{IR}}(E)$ of the IR photons emitted as a function of the internal energy of PAHs, so that one can compute the IR cooling rate as a function of the internal energy of the molecule as

$\frac{\mathrm{d} E}{\mathrm{~d} t}(E)=k_{\mathrm{IR}}(E) \times e_{\mathrm{IR}}(E)$.

Since the values and shape of $k_{\mathrm{IR}}(E)$ were shown to very weakly affect the results of PAH evolution modelling (Le Page et al. $2001)$, both values $k_{\mathrm{IR}}(E)$ and $e_{\mathrm{IR}}(E)$ were only computed for cations in a normal hydrogenation state and used for all charge and hydrogenation states.

\subsubsection{Dissociation}

Photodissociation of PAHs has been experimentally and theoretically studied by several teams. Ekern et al. (1998) qualitatively studied PAH photostability and showed that larger and more compact species are more resistant to UV-visible photons, in agreement with previous results (Jochims et al. 1994). The same team determined the branching ratios of the dissociation paths of fluorene cation $\left(\mathrm{C}_{13} \mathrm{H}_{10}^{+}\right)$showing that the successive loss of $\mathrm{H}$-atoms dominates the other paths like $\mathrm{H}_{2}$-loss or $\mathrm{C}_{2} \mathrm{H}_{2}$-loss (Dibben et al. 2001; Szczepanski et al. 2001). The works of Jochims et al. (1994), Ekern et al. (1998), and Banisaukas et al. (2004) strongly suggest that this behaviour is even more pronounced for larger PAHs. The photodissociation rates of naphthalene $\left(\mathrm{C}_{10} \mathrm{H}_{8}^{+}\right)$, anthracene $\left(\mathrm{C}_{14} \mathrm{H}_{10}^{+}\right)$, phenanthrene $\left(\mathrm{C}_{14} \mathrm{H}_{10}^{+}\right)$, and pyrene $\left(\mathrm{C}_{16} \mathrm{H}_{10}^{+}\right)$have been quantitatively determined by Lifshitz and co-workers (Ho et al. 1995; Ling \& Lifshitz 1998; Ling et al. 1995), who combined experimental results with the Rice-Ramsperger-Kassel-Marcus (RRKM) statistical theory. They showed, in addition, that once a first H-atom is lost, the loss of a second H-atom is faster (Ling \& Lifshitz 1998). Based on these results, Le Page et al. (2001) used a simplified version of RRKM in order to compute the dissociation rates of large PAHs, bearing up to $200 \mathrm{C}$-atoms.

The coronene cation $\left(\mathrm{C}_{24} \mathrm{H}_{12}^{+}\right)$has been more specifically studied by Joblin et al. (in prep.) with the Fourier transform-ion cyclotron resonance trap (FT-ICR) experiment PIRENEA. They confirmed that full dehydrogenation occurs before any carbon loss. In addition, the partially dehydrogenated cations bearing an 

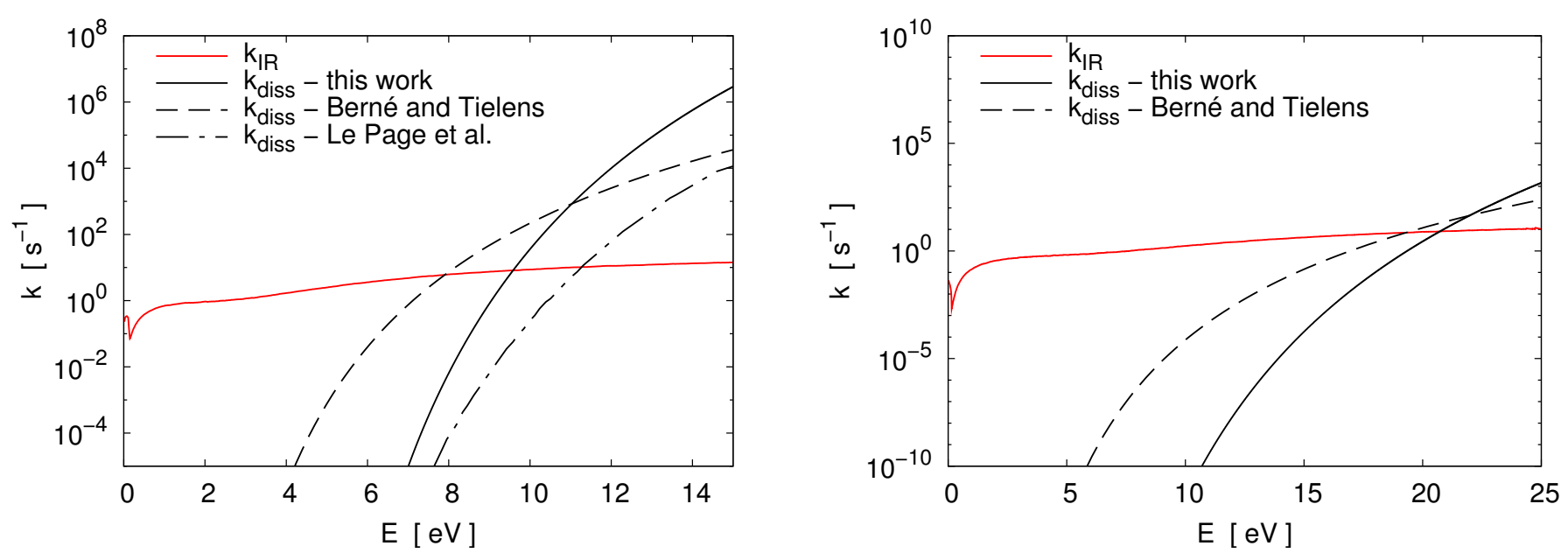

Fig. 3. Rates for IR emission and dissociation as a function of the internal energy for coronene $\mathrm{C}_{24} \mathrm{H}_{12}($ left $)$ and circumovalene $\mathrm{C}_{66} \mathrm{H}_{20}(r i g h t)$. For the typical energies of UV photons in PDRs, our results predict dissociation rates that are much lower than in Berné \& Tielens (2012), and much higher than in Le Page et al. (2001).

odd number of $\mathrm{H}$-atoms are found to photodissociate much faster than those bearing an even number of $\mathrm{H}$-atoms, consistently with the results of Ling \& Lifshitz (1998) on smaller species.

The results of Jochims et al. (1994) and Ling \& Lifshitz (1998) are well explained by statistical theories. This justifies the use of statistical approaches in previous PAH models (Léger et al. 1989; Allain et al. 1996b; Le Page et al. 2001; Visser et al. 2007). We chose to follow the approach described in Léger et al. (1989) and Boissel et al. (1997), based on the Laplace transform of the Arrhenius law:

$k_{\text {diss }}(E)=A_{\text {diss }} \frac{\rho\left(E-E_{0}\right)}{\rho(E)}$

where $E$ is the internal energy of the PAH, $A_{\text {diss }}$ has the dimension of a frequency, $E_{0}$ is the dissociation energy, and $\rho(E)$ is the vibrational density of states of the PAH. This very simple approach was shown to provide satisfying results when compared to more sophisticated methods, like RRKM theory (Barker 1983).

The two parameters $A_{\text {diss }}$ and $E_{0}$ were discussed for the different hydrogenation states of coronene by Joblin et al. (in prep.). The authors show that assuming a dissociation energy $E_{0}=4.8 \mathrm{eV}$ for the $\mathrm{C}-\mathrm{H}$ bond of all PAHs bearing an even number of $\mathrm{H}$-atoms, they manage to account for the experimental results for all these species by using a single value of $A_{\text {diss }}=6.8 \times 10^{17} \mathrm{~s}^{-1}$ that was derived from the photoion appearance potential measured by Jochims et al. (1994) on $\mathrm{C}_{24} \mathrm{H}_{12}$. In addition, the dissociation of PAHs bearing an odd number of $\mathrm{H}$-atoms is described well with the same constant $A_{\text {diss }}$ by assuming $E_{0}=3.2 \mathrm{eV}$. Since there is no data available for the other species, we use the same values of $A_{\text {diss }}$ and $E_{0}$ in the following for the four PAHs considered in this work in their cationic and neutral states. Therefore, the evolution of $k_{\text {diss }}$ with molecular size is driven by the dependence of the vibrational densities of states on size, which basically reflects the evolution of the number of degrees of freedom. We computed the exact quantum harmonic vibrational densities of states with the method of Beyer \& Swinehart (Stein \& Rabinovitch 1973).

Data concerning the dissociation of superhydrogenated PAHs are scarce. They are thought to be more easily dissociated than molecules in a lower hydrogenation state (Le Page et al. 2001, and references therein). Therefore, in the following, we use the dissociation rate of the corresponding PAH missing a single $\mathrm{H}$-atom as a lower limit for their dissociation rate (e.g. $\left.k_{\text {diss }}\left(\mathrm{C}_{24} \mathrm{H}_{13}\right)=k_{\text {diss }}\left(\mathrm{C}_{24} \mathrm{H}_{11}\right)\right)$, leading to an upper limit on the abundances of superhydrogenated species in our results.

Compared to other studies, our work provides significantly different values. The new experimental constraints on coronene cations lead to much more fragile species than predicted by Le Page et al. (2001) (see Fig. 3). In contrast, the more empirical method that is presented in Tielens (2005) and used in Berné $\&$ Tielens (2012) leads to very different values and shapes of $k_{\text {diss }}(E)$ and to a lower stability than for our values. We expect our dissociation rates to provide better results because they are based on experimental data on larger PAHs than considered in previous studies.

\subsection{Reactivity with hydrogen}

Only a few experimental studies of the reactivity of gas-phase PAHs with hydrogen have been published, and all of them treat small cations bearing up to $24 \mathrm{C}$-atoms. Therefore, we first evaluate the reaction rates for coronene cations in all their hydrogenation states from the available data, and then extrapolate these values for the larger PAHs considered in our work. The values of reaction rates for $\mathrm{C}_{24} \mathrm{H}_{N}^{+}$and their origin are summarised in Table 3.

Reactions between PAHs and atomic or molecular hydrogen generally lead to the addition of one or two H-atoms to peripheral C-atoms of the PAH (see discussion by Le Page et al. 2001). The reactivity of PAH cations with atomic hydrogen is found to be rather fast and to decrease when PAH size increases: $2.3 \times 10^{-10} \mathrm{~cm}^{3} \mathrm{~s}^{-1}$ for benzene $\left(\mathrm{C}_{6} \mathrm{H}_{6}^{+}\right.$, Petrie et al. 1992), $1.9 \times 10^{-10} \mathrm{~cm}^{3} \mathrm{~s}^{-1}$ for naphthalene $\left(\mathrm{C}_{10} \mathrm{H}_{8}^{+}\right.$, Snow et al. 1998), $1.4 \times 10^{-10} \mathrm{~cm}^{3} \mathrm{~s}^{-1}$ for pyrene $\left(\mathrm{C}_{16} \mathrm{H}_{10}^{+}\right.$, Le Page et al. 1999a), and $1.4 \times 10^{-10} \mathrm{~cm}^{3} \mathrm{~s}^{-1}$ for coronene $\left(\mathrm{C}_{24} \mathrm{H}_{12}^{+}\right.$, Betts et al. 2006).

Following Le Page et al. (2001), we use a reaction rate for normally hydrogenated PAH cation $\mathrm{C}_{x} \mathrm{H}_{y}^{+}$that scales with the reaction rate of coronene, according to $k_{x, y}=(y / x) k_{\text {coronene. }}$. This expression is in reasonable agreement with available experimental results considering a typical experimental uncertainty of 50\% (Betts et al. 2006). We also follow Le Page et al. (2001) to estimate reaction rates for dehydrogenated and superhydrogenated PAHs, assuming that (i) dehydrogenated coronene 
Table 3. Summary of the measured and inferred reaction rates of atomic and molecular hydrogen with coronene cations in their various hydrogenation states.

\begin{tabular}{|c|c|c|c|c|}
\hline Reaction & $k\left[\mathrm{~cm}^{3} \mathrm{~s}^{-1}\right]$ & Ref. & Comments & Evolution with $N_{\mathrm{C}}$ \\
\hline $\begin{array}{l}\mathrm{C}_{24} \mathrm{H}_{12}^{+}+\mathrm{H} \\
\mathrm{C}_{24} \mathrm{H}_{12-2 n}^{+}+\mathrm{H} \\
\mathrm{C}_{24} \mathrm{H}_{12-2 n+1}^{+}+\mathrm{H} \\
\mathrm{C}_{24} \mathrm{H}_{12+n}^{+}+\mathrm{H}\end{array}$ & $\begin{array}{l}1.4 \times 10^{-10} \\
1.4 \times 10^{-10} \\
\sim 5 \times 10^{-11} \\
\sim 10^{-12}\end{array}$ & $\begin{array}{l}\text { (a) } \\
(\mathrm{b}, \mathrm{c}, \mathrm{d}) \\
(\mathrm{b}, \mathrm{c}) \\
(\mathrm{e})\end{array}$ & $\begin{array}{c}\text { FA-SIFT } \\
\text { extrapolation from } \mathrm{C}_{10} \mathrm{H}_{6}^{+} \text {and } \mathrm{C}_{16} \mathrm{H}_{8}^{+} \\
\text {extrapolation from } \mathrm{C}_{10} \mathrm{H}_{7}^{+} \\
\text {extrapolation from } \mathrm{C}_{6} \mathrm{H}_{7}^{+}, \mathrm{C}_{10} \mathrm{H}_{9}^{+} \text {and } \mathrm{C}_{16} \mathrm{H}_{11}^{+}\end{array}$ & $\begin{array}{c}y / x \\
y / x \\
\text { constant } \\
\text { constant } \\
\end{array}$ \\
\hline $\begin{array}{l}\mathrm{C}_{24} \mathrm{H}_{12}^{+}+\mathrm{H}_{2} \\
\mathrm{C}_{24} \mathrm{H}_{12-2 n}^{+}+\mathrm{H}_{2} \\
\mathrm{C}_{24} \mathrm{H}_{12-2 n+1}^{+}+\mathrm{H}_{2} \\
\mathrm{C}_{24} \mathrm{H}_{12+n}^{+}+\mathrm{H}_{2}\end{array}$ & $\begin{array}{l}<5 \times 10^{-13} \\
<5 \times 10^{-13} \\
\quad- \\
\quad-\end{array}$ & $\begin{array}{l}\text { (a) } \\
\text { (b) } \\
- \\
-\end{array}$ & $\begin{array}{c}\text { FA-SIFT } \\
\text { extrapolation from } \mathrm{C}_{10} \mathrm{H}_{6}^{+} \text {and } \mathrm{C}_{16} \mathrm{H}_{8}^{+} \\
- \\
-\end{array}$ & $\begin{array}{c}\text { constant } \\
\text { constant } \\
- \\
-\end{array}$ \\
\hline
\end{tabular}

Notes. The type of experimental set-up used to perform the measurements is given in the "Comments" column. When "extrapolation" is mentioned, the rate value was extrapolated from measurements on smaller species. FA-SIFT: flowing afterglow-selected ion flow tube. The last column mentions the kind of variation in the rates with the size of PAHs that we considered in our model. When $y / x$ is mentioned, the rate varies proportionately to the ratio of peripheral $(y)$ to total $(x)$ C-atoms in the species.

References. (a) Betts et al. (2006); (b) Le Page et al. (2001); (c) Le Page et al. (1999a); (d) Le Page et al. (1999b); (e) Snow et al. (1998).

cations missing an even number of $\mathrm{H}$-atoms react with the same rate than the normally hydrogenated coronene cation, as inferred from the behaviour of naphthalene and pyrene dehydrogenated cations; (ii) dehydrogenated coronene cations missing an odd number of $\mathrm{H}$-atoms react with a lower rate of $\sim 5 \times$ $10^{-11} \mathrm{~cm}^{3} \mathrm{~s}^{-1}$ as for $\mathrm{C}_{10} \mathrm{H}_{7}^{+}$; (iii) super-hydrogenated coronene has a lower rate of $\sim 10^{-12} \mathrm{~cm}^{3} \mathrm{~s}^{-1}$, as suggested by measurements on smaller PAHs (Snow et al. 1998). The reaction rates for the other PAH species are derived by using a similar scaling for even-dehydrogenated PAHs to those for normally hydrogenated PAHs. No evolution of reaction rates with size is considered for odd-dehydrogenated PAHs and superhydrogenated PAHs.

The reaction rates of PAH cations with molecular hydrogen were determined to be below the experimental detection threshold, which led to an upper limit of $\sim 5 \times 10^{-13} \mathrm{~cm}^{3} \mathrm{~s}^{-1}$ for the reaction rate of $\mathrm{C}_{24} \mathrm{H}_{12}^{+}+\mathrm{H}_{2}$ (Betts et al. 2006). We therefore calculated upper and lower limits to the abundances of PAH hydrogenation states by assuming either that PAHs do not react with $\mathrm{H}_{2}$ or that $\mathrm{PAH}$ cations react with $\mathrm{H}_{2}$ with a reaction rate of $5 \times 10^{-13} \mathrm{~cm}^{3} \mathrm{~s}^{-1}$ leading to the addition of two $\mathrm{H}$-atoms to peripheral sites.

The reactivity of neutral PAHs with atomic hydrogen is unknown. Mebel et al. (1997) theoretically showed the absence of a potential barrier for the reaction $\mathrm{C}_{6} \mathrm{H}_{6}+\mathrm{H}$, suggesting that reaction rates may be high. However, Le Page et al. (2001) find that the results were not affected when including a reaction rate of neutral PAHs with atomic hydrogen of $10^{-10} \mathrm{~cm}^{3} \mathrm{~s}^{-1}$. In our standard model, we neglect the reactivity of neutral PAHs, and afterwards evaluate the uncertainty resulting from this choice, arbitrarily assuming reaction rates of $3 \times 10^{-11} \mathrm{~cm}^{3} \mathrm{~s}^{-1}$ at $300 \mathrm{~K}$ for radical neutral PAHs (odd number of $\mathrm{H}$-atoms), and $3 \times$ $10^{-13} \mathrm{~cm}^{3} \mathrm{~s}^{-1}$ at $300 \mathrm{~K}$ for closed-shell neutral PAHs (even number of $\mathrm{H}$-atoms).

\subsection{Recombination with electrons}

Recombination of PAH cations with electrons leads to an increase in the PAH internal energy by the value of the ionization potential. The statistical approach presented in Sect. 3.3.2 can then be applied to determine whether this extra energy is relaxed radiatively or triggers molecular dissociation. According to the rates shown in Fig. 3 in the case of coronene, IR photon emission is at least 100 times faster than dissociation for an internal energy of $7 \mathrm{eV}$, and the recombination of coronene cations with electrons is therefore expected to be non-dissociative. Larger species have lower values of their ionization potentials and are stabler than coronene. We can therefore neglect the dissociative channel for all the species studied here.

The recombination rates of $\mathrm{PAH}$ cations with electrons were measured for a number of small PAHs (Abouelaziz et al. 1993; Hassouna et al. 2003; Rebrion-Rowe et al. 2003; Novotny et al. 2005; Biennier et al. 2006). All the experimental values are about one order of magnitude lower than the Spitzer law, which is based on the classical model of a thin conducting disk (Spitzer 1978; Verstraete et al. 1990); however, this difference decreases when the size of PAHs increases. To our knowledge, there is no published model available that accurately accounts for this trend. Therefore, for the four PAHs of this study, we assume that the recombination rates are comprised between the experimental rate of pyrene cations and the Spitzer law, so we use the average of these two values in our model. This leads to rates that are roughly one order of magnitude higher than those proposed by Le Page et al. (2001).

\subsection{Charge exchange}

Charge exchange reactions between neutral PAHs and ions such as $\mathrm{C}^{+}, \mathrm{Fe}^{+}$, or $\mathrm{Si}^{+}$may impact the ionization balance of PAHs. Reactions with $\mathrm{C}^{+}$proceed at the Langevin rate (Canosa et al. 1995), and can marginally affect the PAH charge state in low UV irradiation environments like the diffuse ISM (Wolfire et al. 2008). Photoionization rapidly dominates the ionization balance of PAHs when the UV radiation field intensity increases. Other possible partners for charge exchange are much less abundant than $\mathrm{C}^{+}$and are therefore completely negligible. In the following, we do not consider charge exchange reactions. We check the validity of this assumption in Sect. 5.3.

\subsection{Sum-up of our standard model}

The species and processes included in our model are summarised in Table 4. In short, we consider here four sizes of PAHs in various hydrogenation states and two charge states, namely coronene $\left(\mathrm{C}_{24} \mathrm{H}_{N}^{0 /+}\right)$, circumcoronene $\left(\mathrm{C}_{54} \mathrm{H}_{N}^{0 /+}\right)$, circumovalene $\left(\mathrm{C}_{66} \mathrm{H}_{N}^{0 /+}\right)$, and circumcircumcoronene $\left(\mathrm{C}_{96} \mathrm{H}_{N}^{0 /+}\right)$. We limit the possible charge states to neutrals and monocations, excluding dications and anions for several converging reasons, including (i) the lack of data for the recombination of dications with electrons 
Table 4. Species and main parameters of the processes considered in our standard model.

\begin{tabular}{|c|c|c|c|c|c|c|c|c|c|c|}
\hline & $N_{\mathrm{C}}$ & $N_{\mathrm{H}}^{0}$ & $q$ & $N_{\mathrm{H}}$ & $\begin{array}{c}\text { IP } \\
{[\mathrm{eV}]}\end{array}$ & $\begin{array}{c}\alpha(300 \mathrm{~K}) \\
{\left[\mathrm{cm}^{3} \mathrm{~s}^{-1}\right]}\end{array}$ & $\begin{array}{c}E_{0} \\
{[\mathrm{eV}]}\end{array}$ & $\begin{array}{l}A_{\text {diss }} \\
{\left[\mathrm{s}^{-1}\right]}\end{array}$ & $\begin{array}{c}k_{+\mathrm{H}}(300 \mathrm{~K}) \\
{\left[\mathrm{cm}^{3} \mathrm{~s}^{-1}\right]}\end{array}$ & $\begin{array}{c}k_{+\mathrm{H}_{2}} \\
{\left[\mathrm{~cm}^{3} \mathrm{~s}^{-1}\right]}\end{array}$ \\
\hline Ho & 24 & 12 & 0 & $\begin{array}{c}2 n \\
2 n+1 \\
13 \\
2 n \\
2 n+1 \\
13\end{array}$ & $\begin{array}{l}7.02 \\
7.02 \\
7.02 \\
- \\
- \\
- \\
\end{array}$ & $\begin{array}{c}- \\
- \\
- \\
1.0 \times 10^{-5} \\
1.0 \times 10^{-5} \\
1.0 \times 10^{-5}\end{array}$ & $\begin{array}{l}4.8 \\
3.2 \\
3.2 \\
4.8 \\
3.2 \\
3.2 \\
\end{array}$ & $6.8 \times 10^{17}$ & $\begin{array}{c}0 / 3 \times 10^{-13} \\
0 / 3 \times 10^{-11} \\
0 / 3 \times 10^{-13} \\
1.4 \times 10^{-10} \\
5 \times 10^{-11} \\
-\end{array}$ & $\begin{array}{c}0 \\
0 \\
0 \\
0 / 5 \times 10^{-13} \\
0 / 5 \times 10^{-13} \\
-\end{array}$ \\
\hline $\begin{array}{l}001 \\
200\end{array}$ & 54 & 18 & 0 & $\begin{array}{c}2 n \\
2 n+1 \\
19 \\
2 n \\
2 n+1 \\
19\end{array}$ & $\begin{array}{l}6.14 \\
6.14 \\
6.14 \\
- \\
- \\
-\end{array}$ & $\begin{array}{c}- \\
- \\
- \\
1.4 \times 10^{-5} \\
1.4 \times 10^{-5} \\
1.4 \times 10^{-5}\end{array}$ & $\begin{array}{l}4.8 \\
3.2 \\
3.2 \\
4.8 \\
3.2 \\
3.2\end{array}$ & $6.8 \times 10^{17}$ & $\begin{array}{c}0 / 3 \times 10^{-13} \\
0 / 3 \times 10^{-11} \\
0 / 3 \times 10^{-13} \\
9.3 \times 10^{-11} \\
5 \times 10^{-11} \\
-\end{array}$ & $\begin{array}{c}0 \\
0 \\
0 \\
0 / 5 \times 10^{-13} \\
0 / 5 \times 10^{-13} \\
-\end{array}$ \\
\hline wio & 66 & 20 & 0 & $\begin{array}{c}2 n \\
2 n+1 \\
21 \\
2 n \\
2 n+1 \\
21\end{array}$ & $\begin{array}{l}5.71 \\
5.71 \\
5.71 \\
- \\
- \\
-\end{array}$ & $\begin{array}{c}- \\
- \\
- \\
1.56 \times 10^{-5} \\
1.56 \times 10^{-5} \\
1.56 \times 10^{-5}\end{array}$ & $\begin{array}{l}4.8 \\
3.2 \\
3.2 \\
4.8 \\
3.2 \\
3.2\end{array}$ & $6.8 \times 10^{17}$ & $\begin{array}{c}0 / 3 \times 10^{-13} \\
0 / 3 \times 10^{-11} \\
0 / 3 \times 10^{-13} \\
8.5 \times 10^{-11} \\
5 \times 10^{-11} \\
-\end{array}$ & $\begin{array}{c}0 \\
0 \\
0 \\
0 / 5 \times 10^{-13} \\
0 / 5 \times 10^{-13} \\
-\end{array}$ \\
\hline $\begin{array}{l}606 \\
.\end{array}$ & 96 & 24 & 0 & $\begin{array}{c}2 n \\
2 n+1 \\
25 \\
2 n \\
2 n+1 \\
25\end{array}$ & $\begin{array}{l}5.68 \\
5.68 \\
5.68 \\
- \\
- \\
-\end{array}$ & $\begin{array}{c}- \\
- \\
- \\
1.85 \times 10^{-5} \\
1.85 \times 10^{-5} \\
1.85 \times 10^{-5}\end{array}$ & $\begin{array}{l}4.8 \\
3.2 \\
3.2 \\
4.8 \\
3.2 \\
3.2\end{array}$ & $6.8 \times 10^{17}$ & $\begin{array}{c}0 / 3 \times 10^{-13} \\
0 / 3 \times 10^{-11} \\
0 / 3 \times 10^{-13} \\
7.0 \times 10^{-11} \\
5 \times 10^{-11} \\
-\end{array}$ & $\begin{array}{c}0 \\
0 \\
0 \\
0 / 5 \times 10^{-13} \\
0 / 5 \times 10^{-13} \\
-\end{array}$ \\
\hline
\end{tabular}

Notes. The columns give: (1) the structure of the species studied in this paper in their neutral normally hydrogenated state; (2) the number $N_{\mathrm{C}}$ of C-atoms in the species; (3) the number $N_{\mathrm{H}}^{0}$ of H-atoms in the normally hydrogenated state; (4) the charge $q$ of the species; (5) the number $N_{\mathrm{H}}$ of H-atoms in the species ( $2 n$ is for normally hydrogenated PAHs and PAHs missing an even number of H-atoms, $2 n+1$ is for PAHs missing an even number of $\mathrm{H}$-atoms; other values are superhydrogenated states); (6) the ionization potential IP of the species; (7) the rate of recombination $\alpha$ with electrons at $300 \mathrm{~K} ;(8)$ the binding energy $E_{0} ;(9)$ the pre-exponential factor $A_{\text {diss }}$ used in Eq. (3); (10) the reaction rate for $\mathrm{H}$-atom addition at $300 \mathrm{~K}$; (11) the rate for reaction with $\mathrm{H}_{2}$. For (10) and (11), when two values are given, they correspond to the lower/upper limits considered in our model.

and for the association of neutral PAHs with electrons, and (ii) the expected weak abundances of these other charge states in the environments considered here. The latter point can be checked afterwards. We consider all the hydrogenation states from fully dehydrogenated species, i.e. pure carbon clusters, to superhydrogenated PAHs bearing one extra $\mathrm{H}$-atom. This choice is motivated by the lack of quantitative data for both the dissociation rates of superhydrogenated species and the rates of reaction of these species with hydrogen. The dissociation rate that is used for superhydrogenated PAHs should be considered as a lower limit.

The charge evolution is driven by the balance between photoionization and recombination with electrons, in our model, both depending exclusively on the size of PAHs. Similarly, the hydrogenation state is driven by the balance between photodissociation and reactivity with atomic and molecular hydrogen. Photodissociation strongly depends on the size of the species through their vibrational density of states, and also strongly on their hydrogenation state through the binding energy $E_{0}$, PAHs with an odd number of $\mathrm{H}$ being less stable than PAHs with an even number of H. Dependence on the charge state is not considered in our model for this process. In constrast, reactivity with atomic and molecular hydrogen mainly depends on the charge and, to a lesser extent, on the hydrogenation state of PAHs, and more marginally on their size. Considering the lack of data for the reaction rates of PAH cations with $\mathrm{H}_{2}$ and of neutral PAHs with $\mathrm{H}$, we consider lower and upper values for these processes.
Describing PAH evolution requires modelling the processes discussed above, as well as considering how they vary with astrophysical conditions. The UV-visible radiation field spectrum drives both photoionization and photodissociation. The abundances of $\mathrm{H}, \mathrm{H}_{2}$, and free electrons are involved through their reactions with PAHs, in which the gas kinetic temperature $T$ plays a role. Considering only the influence of temperature on the collision rates, the rates of recombination with electrons vary like $T^{-1 / 2}$ (see, e.g., Bakes \& Tielens 1994), the rates of reaction between two neutral species like $T^{1 / 2}$, and no variation with $T$ is considered for reactions between charged and neutral species (Le Page et al. 2001; Herbst 2001, and references therein).

\section{Numerical framework}

\subsection{Description of the species}

The species considered in this work are presented in Sect. 3.7 and Table 4. The evolution of these species involves some processes that critically depend on the internal energy of the reactants, like photodissociation processes. An explicit description of this internal energy was proposed by Visser et al. (2007) in the context of protoplanetary disks, where the strong UV-visible irradiation by the close illuminating star is expected to favour multiphoton events, i.e. absorption of another photon before complete cooling of the PAHs. Previous studies of the evolution of PAHs in regions of low or moderate excitation do not 

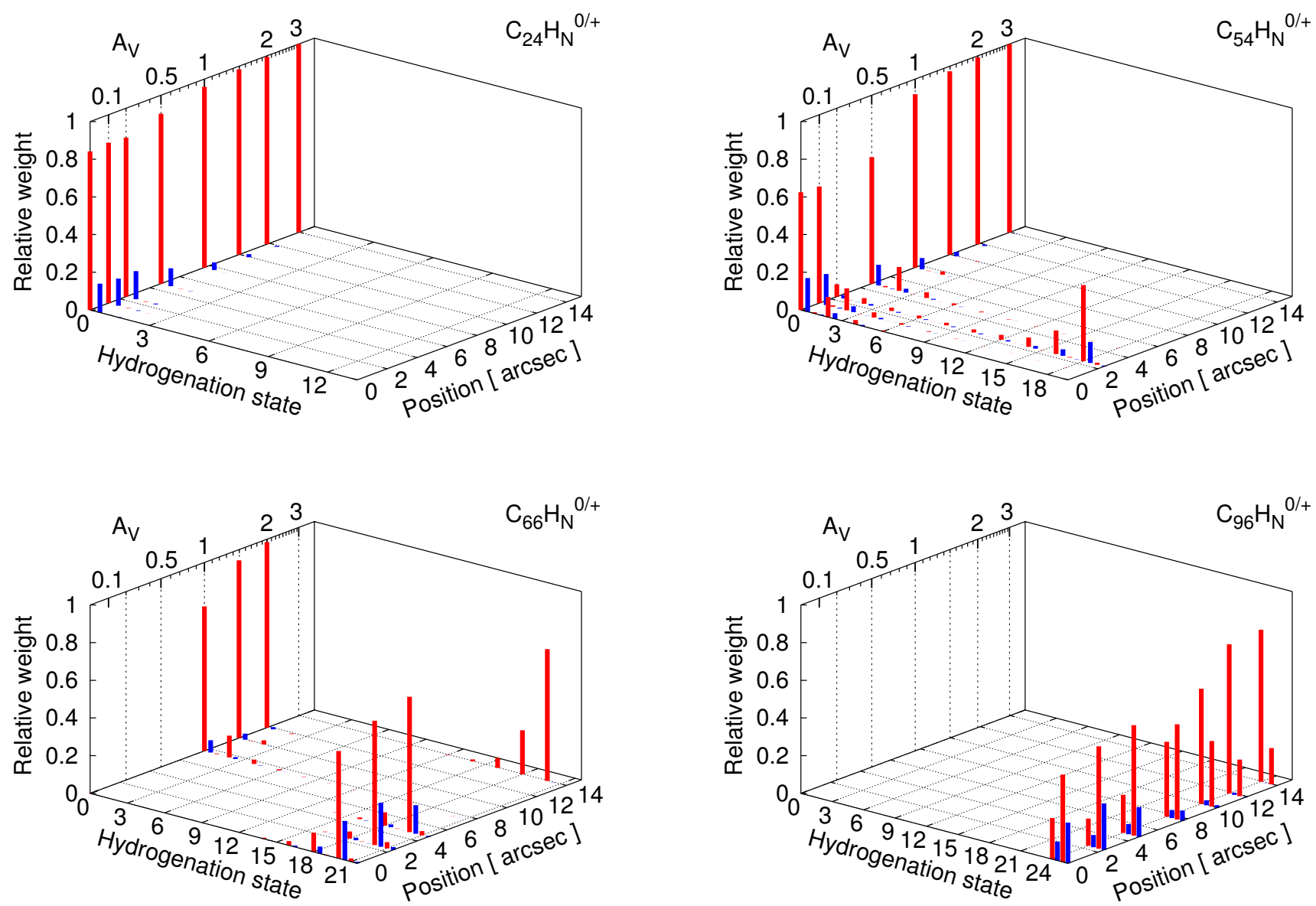

Fig. 4. Charge and hydrogenation states (number of $\mathrm{H}$-atoms in the $\mathrm{PAH})$ of coronene $\left(\mathrm{C}_{24} \mathrm{H}_{12}\right)$, circumcoronene $\left(\mathrm{C}_{54} \mathrm{H}_{18}\right)$, circumovalene $\left(\mathrm{C}_{66} \mathrm{H}_{20}\right)$, and circumcircumcoronene $\left(\mathrm{C}_{96} \mathrm{H}_{24}\right)$ as a function of the position in the NW PDR of NGC 7023, with the astrophysical conditions presented in Fig. 2 and with $k_{+\mathrm{H}_{2}}=0$ and $k_{+\mathrm{H}}$ (neutral) $=0$. Neutral species are in red, cations in blue.

explicitly describe the evolution of the internal energy of PAHs (Allain et al. 1996a; Le Page et al. 2001; Berné \& Tielens 2012). Nevertheless, a quick examination of the rates for IR photon emission $\left(k_{\mathrm{IR}}\right)$ and dissociation $\left(k_{\mathrm{diss}}\right.$, see Fig. 3) reveals that for large enough species, the threshold energy $E_{\mathrm{th}}$ for which $k_{\mathrm{IR}}\left(E_{\mathrm{th}}\right)=k_{\mathrm{diss}}\left(E_{\mathrm{th}}\right)$ falls well above the Lyman cut-off at $13.6 \mathrm{eV}$. As a consequence, even in low radiation fields, dissociation is driven by multiphoton events, since this is the only way to raise the internal energy of large PAHs close to or above $E_{\mathrm{th}}$.

To describe the evolution of internal energy, each species $X$ was divided into an ensemble of subspecies $X_{i}(i=0, \ldots$, $N_{\text {bin }}$ ) whose number density is the number per unit of volume of species $X_{i}$ bearing an internal energy between $(i-1) \times \Delta E$ and $i \times \Delta E$ for $\left(i=1, \ldots, N_{\mathrm{bin}}\right)$ or no internal energy for $i=0$. Thus, instead of studying the evolution of an ensemble of species, we study the evolution of the histograms of their internal energy. Obviously, the accuracy of the method depends on the width of the bins, $\Delta E$, that we found to be optimum for $\Delta E=0.25 \mathrm{eV}$, which is a compromise between accuracy and computing time (see Appendix A.4).

\subsection{Evolutionary scheme}

We aim at computing the time evolution of the species defined in the previous section in a specific environment. The feedback of PAH evolution on their astrophysical environment is beyond the scope of this work. Therefore, the densities of $\mathrm{H}, \mathrm{H}_{2}$ and free electron are considered as fixed external parameters, as are the kinetic gas temperature and the UV-visible flux. We compute the time evolution of each species $X$ and its internal energy histograms by considering the chemical balance of each subspecies $X_{i}$ :

$\frac{\partial\left[X_{i}\right]}{\partial t}=P_{i}-L_{i}$

where $\left[X_{i}\right]$ is the number density of the species $X_{i}$, and $P_{i}$ and $L_{i}$ are the production and loss rates, respectively. These two latter quantities are computed from (i) the conditions of the astrophysical environment, and (ii) the rates of the processes presented in Sect. 3. Our work differs from previous studies by the way we model photodissociation processes. Instead of computing an effective photodissociation rate for each species $X$, we compute the production and loss rates for each subspecies $X_{i}$ according to the following scheme:

$$
\begin{aligned}
& X_{i}+h v_{\mathrm{UV}} \stackrel{\left[1-Y_{\mathrm{ion}}(i)\right] k_{\mathrm{abs}}(i)}{\longrightarrow} X_{i^{\prime}} \\
& X_{i} \stackrel{k_{\mathrm{IR}}(i)}{\longrightarrow} \quad X_{i^{\prime \prime}}+h v_{\mathrm{IR}} \\
& X_{i} \stackrel{k_{\mathrm{diss}}(i)}{\longrightarrow} \quad X_{0}^{-\mathrm{H}}+\mathrm{H}
\end{aligned}
$$



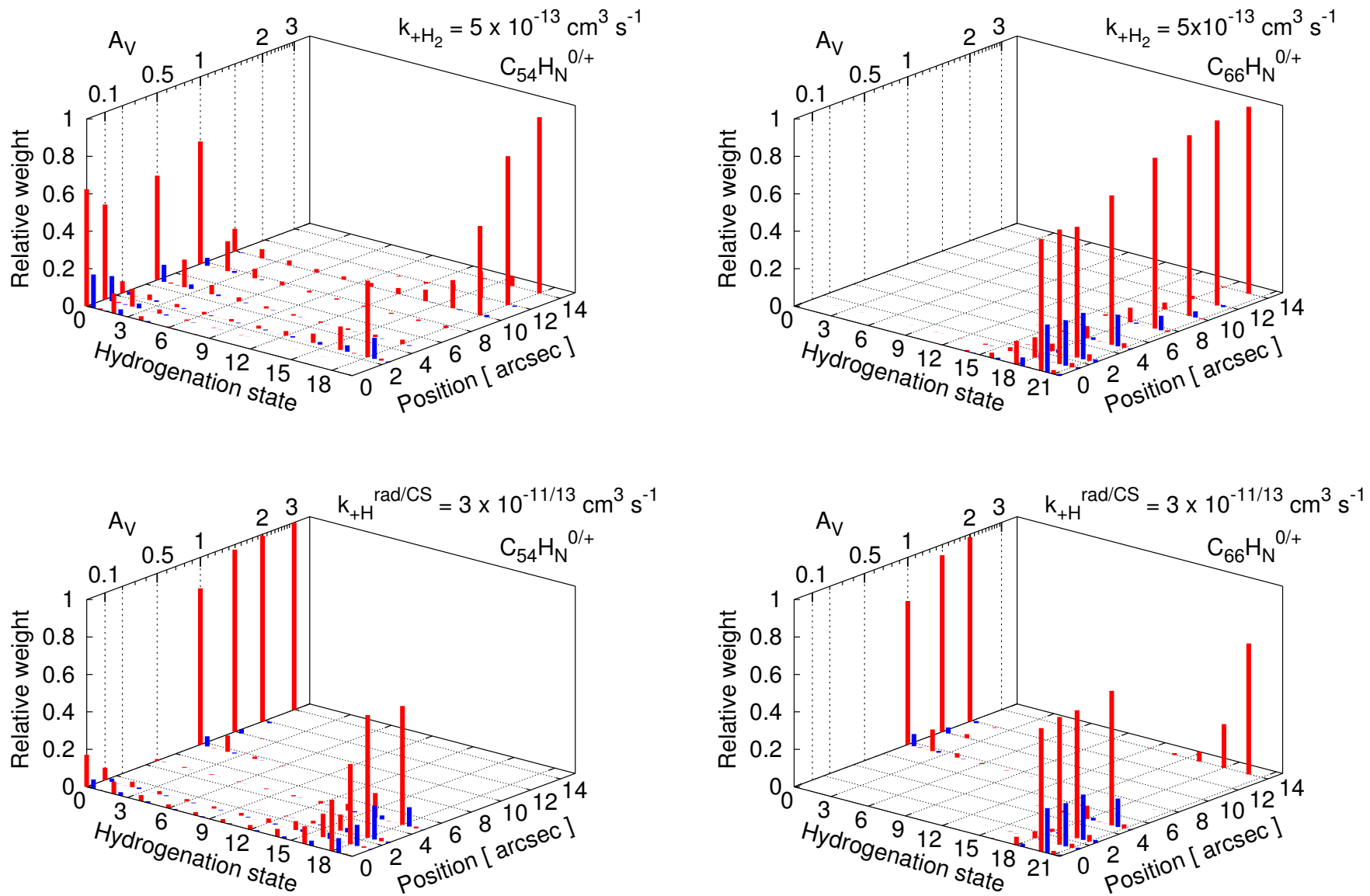

Fig. 5. Same as Fig. 4 but with a reactivity of PAH cations with $\mathrm{H}_{2}$ of $k_{+\mathrm{H}_{2}}=5 \times 10^{-13} \mathrm{~cm}^{3} \mathrm{~s}^{-1}$ (upper panel), and with a reactivity of neutral PAHs with $\mathrm{H}$ of $k_{+\mathrm{H}}^{\text {rad }}=3 \times 10^{-11} \mathrm{~cm}^{3} \mathrm{~s}^{-1}$ for radicals and $k_{+\mathrm{H}}^{\mathrm{CS}}=3 \times 10^{-13} \mathrm{~cm}^{3} \mathrm{~s}^{-1}$ for closed-shell (lower panel). Figures for coronene $\left(\mathrm{C}_{24} \mathrm{H}_{N}^{+/ 0}\right.$ ) and circumcircumcoronene $\left(\mathrm{C}_{96} \mathrm{H}_{N}^{+/ 0}\right)$ are not shown since they exhibit only marginal variations with respect to Fig. 4 . Neutral species are in red, cations in blue.

where $i^{\prime}>i, i^{\prime \prime}<i$, and $X_{0}^{-\mathrm{H}}$ has no internal energy. The numerical rates $\left[1-Y_{\mathrm{ion}}(i)\right] k_{\mathrm{abs}}(i), k_{\mathrm{IR}}(i)$ and $k_{\mathrm{diss}}(i)$ are computed from the physical quantities $Y_{\text {ion }}(E), \sigma_{\text {abs }}(E)$, the UV-visible flux, $k_{\mathrm{IR}}(E)$ and $k_{\mathrm{diss}}(E)$, as detailed in Appendix A. For the other processes, we do not take the effects of the internal energy of PAHs into account, which makes the numerical implementation straightforward.

\section{Results}

The computed charge and hydrogenation states of coronene, circumcoronene, circumovalene and circumcircumcoronene in the NGC 7023 NW PDR are shown in Figs. 4 and 5 for different assumptions concerning the reactivity of PAHs with hydrogen. For all our calculations, we assumed neutral normally hydrogenated PAHs as initial conditions. In this section, we present our results by focussing successively on the charge state and the hydrogenation state of PAHs once steady state is reached.

\subsection{Evolution of PAH charge in NGC 7023-NW}

Using our model, we computed the global cation fraction for the four PAHs considered in this study by summing the cation fraction in all hydrogenation states (e.g. for coronene $\sum_{N_{\mathrm{H}}} \mathrm{C}_{24} \mathrm{H}_{N_{\mathrm{H}}}^{+} /$ $\sum_{N_{\mathrm{H}}, q} \mathrm{C}_{24} \mathrm{H}_{N_{\mathrm{H}}}^{q}$ with $\left.N_{\mathrm{H}}=[0,12]\right)$. In Fig. 6 , the calculated values are compared with the cation fraction extracted from the analysis of the AIB spectrum along the Star-NW cut (Sect. 2).

The computed cation fractions are in good agreement with the observed one in the cavity, as we predict fractions above $90 \%$ of cations at $20^{\prime \prime}$ from the star. In the region of the PDR where ionization of PAHs is found to be significant $\left(A_{V} \lesssim 2\right)$, photoionization dominates the evolution of the charge state of PAHs since the UV radiation field intensity varies by a factor $\sim 10$, whereas the PDR model predicts a rather constant abundance of free electrons and a gas temperature that changes by a factor of 2 (see Fig. 2), which results in a variation in the rate of recombination of PAH cations with free electrons by a factor of less than 2 . The agreement between computed and observed cation fractions is also good within the AIB peak emission region (between 42" and $52^{\prime \prime}$ from the star along the Star-NW cut), in terms of both absolute values and profile evolution. Finally, the cation fraction is found to increase with the PAH size, $N_{\mathrm{C}}=96$ being twice more ionized than $N_{\mathrm{C}}=24$. A more quantitative comparison between modelling and observations would require to weight each calculated charge ratios by the column density of the corresponding PAH along the line of sight.

\subsection{Hydrogenation state}

As shown in Fig. 4, the hydrogenation state of PAHs evolves significantly with both PAH size and position within the PDR. 


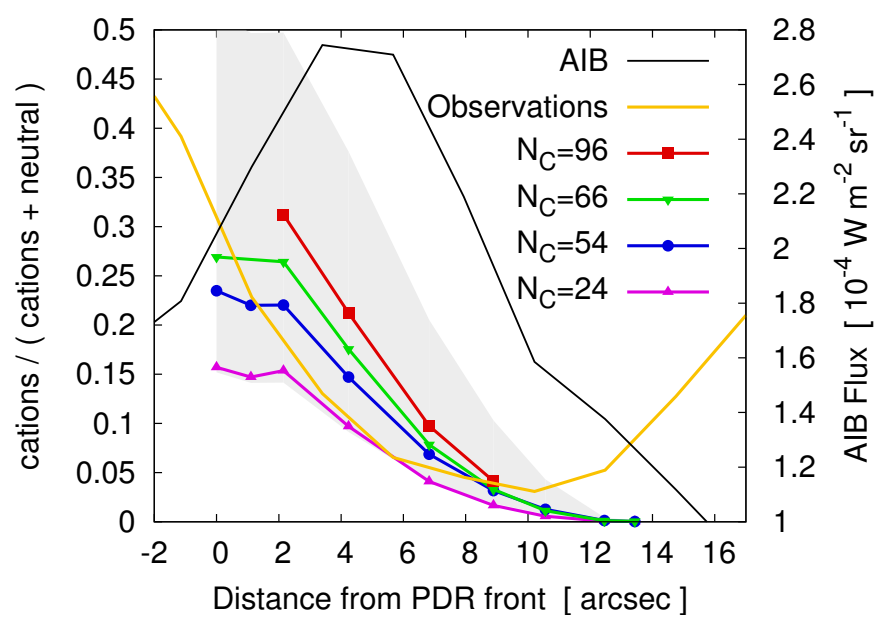

Fig. 6. Fraction of ionized species (PAHs and carbon clusters) along the Star-NW cut in the NW PDR as derived from the observations (yellow, from Pilleri et al. 2012) and as computed with our model (other colours). The total AIB flux is also plotted in black. The grey surface shows the uncertainty range on the ionized fraction for $\mathrm{C}_{54} \mathrm{H}_{N}^{+}$species $(N=0-19)$, which reflects the range of values we considered for the electron recombination rate. The PDR front is assumed to lie at $42^{\prime \prime}$ from the star.

A general result is that partially dehydrogenated species never dominate in terms of abundances. Even collectively, their maximum weight is $40 \%$, and is so for a very narrow range of spatial positions. Therefore, the resulting hydrogenation states can be classified into four categories: pure carbon clusters $\left(N_{\mathrm{H}}=0\right)$, partially dehydrogenated PAHs $\left(1 \leq N_{\mathrm{H}} \leq N_{\mathrm{H}}^{0}-1\right)$, normally hydrogenated PAHs, and superhydrogenated PAHs $\left(N_{\mathrm{H}}=N_{\mathrm{H}}^{0}+1\right)$. This behaviour results from a general trend that more dehydrogenated PAHs are less photostable. Therefore, once a normally hydrogenated PAH starts losing $\mathrm{H}$-atoms, further dehydrogenation is even faster.

We summarise the evolution of the dominant hydrogenation state as a function of the position and size of the species in Fig. 7 (results in the cavity are not shown). To illustrate the contribution of each population to the AIB emission, Fig. 7 also shows $P_{\mathrm{AIB}}$, the emission power per unit volume in the mid-IR domain. It was calculated using the formula $P_{\mathrm{AIB}}=\epsilon n_{\mathrm{H}} G_{0}$ (Pilleri et al. 2012), where $\epsilon=5 \times 10^{-32} \mathrm{~W} \mathrm{H}^{-1}$ and $G_{0}$ is expressed in Habing units. In this formula, all species, whether PAHs or carbon clusters, are assumed to emit the same power per C-atom. For a given position in the PDR, larger molecules tend to be more hydrogenated than smaller ones, as expected. For a given size, however, one alternatively finds hydrogenated and dehydrogenated species when increasing the distance from the star, whereas a monotonous behaviour, as a function of the intensity of the UV radiation field, could be expected. Similarly, superhydrogenated PAHs dominate close to the cloud surface, and are less abundant in deeper layers. These inversion features are due to the decrease in atomic hydrogen density from $A_{V}=0.2$ to $A_{V} \sim 2$, and are therefore a consequence of both the environment evolution and the assumption that reactivity with $\mathrm{H}_{2}$ can be neglected. We discuss this assumption further in Sect. 6.1.

Superhydrogenated PAHs are found to be abundant only for the largest species $\left(N_{\mathrm{C}}=96\right)$, even though we considered a lower limit for the dissociation rate of superhydrogenated PAHs. Pure carbon clusters are found to be the dominant form for small- $\left(N_{\mathrm{C}}<50\right)$ and, possibly, medium-sized $\left(50 \lesssim N_{\mathrm{C}} \lesssim 70\right)$ species, until cloud depths, at which PAHs are thought to be
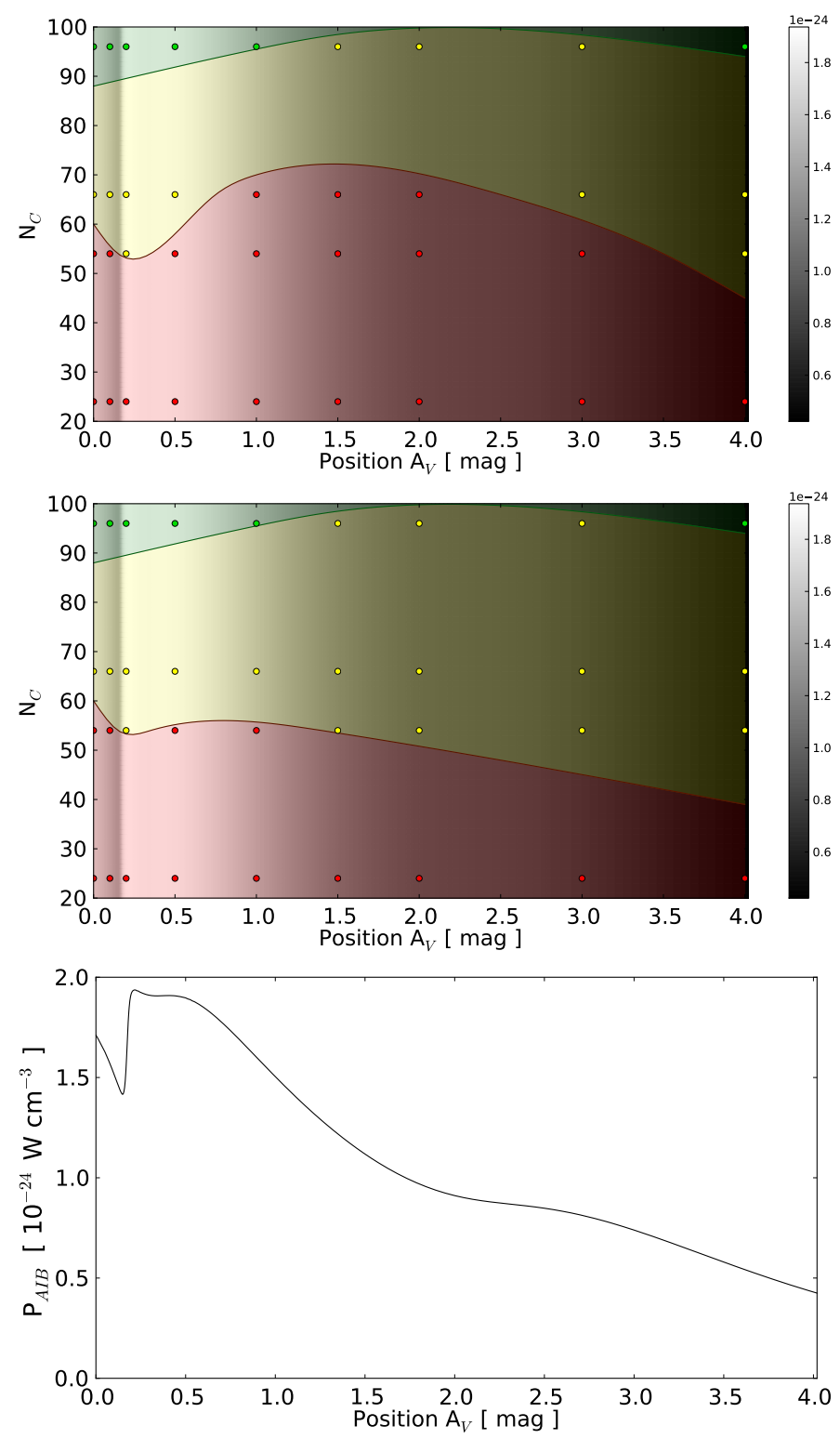

Fig. 7. Dominant hydrogenation state as a function of PAH size and position in the NGC $7023 \mathrm{NW}$ PDR, without reactivity (upper panel) or with a rate of $5 \times 10^{-13} \mathrm{~cm}^{3} \mathrm{~s}^{-1}$ (middle panel) for the reaction between PAH cations and $\mathrm{H}_{2}$. The points represent the parameters for which models were computed. Red, yellow, and green represent carbon clusters, normally hydrogenated PAHs, and superhydrogenated PAHs (limited to one extra $\mathrm{H}$-atom), respectively. Lines delimiting the different domains were interpolated to guide the eye and should be considered with caution. The power per unit of volume $P_{\mathrm{AIB}}$ emitted in the mid-IR domain by PAHs and carbon clusters as a function of their position is shown in the lower panel, and is also overlaid as a brightness scale to the upper and middle panels. The variation observed between $A_{V}=0$ and 0.2 reflects the variation of $n_{\mathrm{H}}$ (see Fig. 2).

part of very small grains $\left(A_{V} \gtrsim 2\right.$, see Fig. 2 and Pilleri et al. 2012). In the cavity, where the density is lower and the radiation field much stronger, we predict all PAHs will be completely dehydrogenated.

Interestingly, circumcoronene and circumovalene are found to be fully dehydrogenated in the NW PDR at $A_{V}=0$, and between $A_{V}=1$ and $A_{V}=2$, respectively, whereas for the same conditions, previous models (Le Page et al. 2001; Berné \& Tielens 2012) would predict that normally hydrogenated 


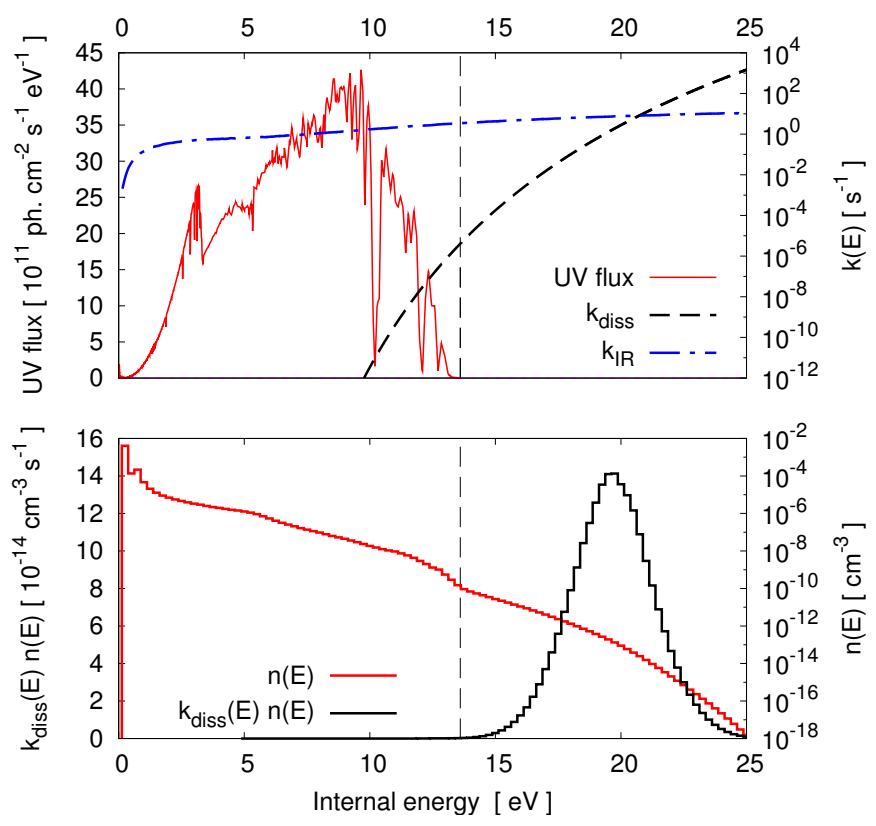

Fig. 8. Upper panel: spectrum of the local UV-visible radiation field in NGC $7023-\mathrm{NW}$ at $42^{\prime \prime}\left(A_{V}=0\right)$ from the star, compared to the rates of dissociation and IR emission of the circumovalene molecule $\mathrm{C}_{66} \mathrm{H}_{20}$. Lower panel: the internal energy of $\mathrm{C}_{66} \mathrm{H}_{20}$ (red histogram) computed with our model in steady state, in the radiation field shown in the upper panel. The black histogram shows the distribution of internal energies of $\mathrm{C}_{66} \mathrm{H}_{20}$ molecules prior to dissociation. The vertical dashed lines show the $13.6 \mathrm{eV}$ limit, emphasising that dissociation is dominated by multiphoton events.

circumovalene would dominate. This discrepancy is due to multiphoton events that are taken into account in our model. Multiphoton events are by far the main channel for PAH dissociation at threshold energy (where IR photon emission and dissociation are equally probable) above the Lyman limit. Figure 8 shows, in the case of circumovalene $\mathrm{C}_{66} \mathrm{H}_{20}$, that cooling by IR photon emission is ten orders of magnitude faster than dissociation at wavelengths of maximum UV radiation. $\mathrm{C}_{66} \mathrm{H}_{20}$ molecules bearing an internal energy close to the threshold energy $(\sim 20 \mathrm{eV})$ after the successive absorption of several UV photons are found to be the most numerous to dissociate, despite their very low abundances (see Fig. 8).

\subsection{Timescales}

The results presented in the previous sections are obtained after steady state has been reached. We investigate here how steady state timescales compare with other evolutionary timescales in a given environment. The steady state for PAH charge evolution is reached within a few hours at the position $A_{V}=1$. As a comparison, for these conditions, the typical timescale for $\mathrm{C}$ photoionization is $\sim 1$ month (Le Petit et al. 2006). This timescale can also be compared with the timescale for charge exchange (c.e.) between neutral PAHs and $\mathrm{C}^{+}$. In the conditions of NGC 7023 $\mathrm{NW}$, using $k_{\text {c.e. }}=3 \times 10^{-9} \mathrm{~cm}^{3} \mathrm{~s}^{-1}$ from Canosa et al. (1995), $n_{\mathrm{H}}=10^{4} \mathrm{~cm}^{3}, \mathrm{C} / \mathrm{H}=1.3 \times 10^{-4}$ from our PDR calculations, and assuming that all atomic carbon is ionized, we find $\tau=1 /\left(k_{\text {c.e. }}\left[\mathrm{C}^{+}\right]\right) \sim 8$ years. This shows that charge exchange is negligible in these conditions.

The steady state for PAH hydrogenation is reached for all species on much longer timescales, which span a very broad range of values depending on $\mathrm{PAH}$ size. Small species reach their steady state within less than a year (e.g. $\sim 1$ month for coronene at $A_{V}=1$ ), while large species exhibit very long timescales, as can be seen in Fig. 9, with $\sim 10^{2}$ years at $A_{V}=0$ and $\sim 10^{4}$ years at $A_{V}=1$ in the case of circumovalene, if no reactivity is considered with $\mathrm{H}_{2}$. When considering the reactivity of PAH cations with $\mathrm{H}_{2}$ at a rate of $k_{+\mathrm{H}_{2}}=5 \times 10^{-13} \mathrm{~cm}^{3} \mathrm{~s}^{-1}$, the timescale at $A_{V}=1$ decreases down to $\sim 10^{2}-10^{3}$ years. These values are very high with respect to the typical hydrogenation time $\left(1 /\left(k_{+\mathrm{H}} \times n_{\mathrm{H}}\right) \sim 10\right.$ days $)$, and seem surprising at first sight. In fact, this is linked to the absence of dominating partially hydrogenated species: the global time evolution for one PAH species scales with the typical times needed for it to evolve through all its partially hydrogenated states, from the normally hydrogenated state to the fully dehydrogenated state and vice versa. These timescales become long when multiphoton events are required to dissociate PAHs, because each dehydrogenation step behaves as a limiting step. This illustrates that the complex evolution of the hydrogenation state of PAHs requires detailed modelling.

Considering that Alecian et al. (2008) estimated the age of HD 200775 as around $10^{5}$ years, our results suggest that the evolution of large PAHs is coupled to the dynamics of the region, and that large PAHs may never reach their steady state abundances.

\section{Sensitivity to parameters}

\subsection{Identifying the key processes}

We have discussed in Sect. 3 the uncertainties on the parameters of the various processes. In this section, we aim at evaluating the sensitivity of the results to some of them, in order to identify the processes that require further investigation.

Determination of the charge state of PAHs is mainly limited by the knowledge of the rate $\alpha$ for their recombination with electrons. In the case of circumcoronene, we computed additional models with the upper and lower values of $\alpha$ as defined in Sect. 3.5. In Fig. 6, we represented the domain of values resulting from this uncertainty. Thus, the fraction of PAH cations is determined with a typical uncertainty of a factor of two, which is slightly more than the dispersion of cation fraction between PAHs of different sizes. This means that further constraints on the PAH size distribution using PAH charge states would require more investigation of the electronic recombination process.

The results presented in Fig. 4 do not consider any reactivity of PAHs with $\mathrm{H}_{2}$, and of neutral PAHs with $\mathrm{H}$. Figure 5 shows the influence of these two processes. The reactivity of neutral PAHs affects the first layers of PDRs, at the interface with the cavity where atomic hydrogen dominates, whereas the reactivity of PAH cations with $\mathrm{H}_{2}$ plays a significant role deeper in the PDR, between $A_{V}=0.5$ and 3 . The species that are smallest in size $\left(N_{\mathrm{C}}=24\right)$ are not affected by these processes since they remain fully dehydrogenated. Similarly, the high photostability of the largest species $\left(N_{\mathrm{C}}=96\right)$ ensures they remain in their normally hydrogenated state regardless of these additional processes. In contrast, intermediate species can be significantly more hydrogenated. The size - position diagrams (Fig. 7) show that the uncertainty on the reaction rates with hydrogen leads to some uncertainty on the position of the transition between normally hydrogenated PAHs and carbon clusters, which lies between $N_{\mathrm{C}} \sim 50$ and $\sim 60$ at $A_{V}=0$ and between $N_{\mathrm{C}} \sim 50$ and $\sim 70$ at $A_{V}=1$. Therefore, the lack of knowledge on these processes, and particularly the reactivity with $\mathrm{H}_{2}$, appears to be a major limitation for predicting the minimum size of PAHs in PDRs. 

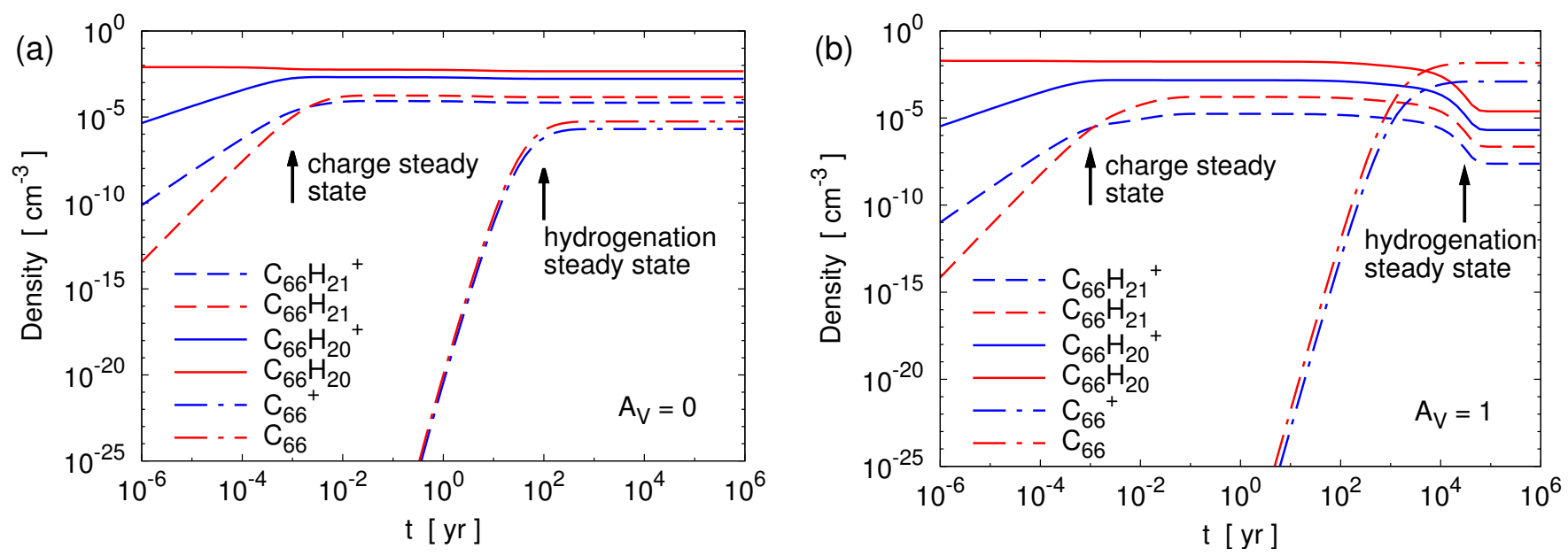

Fig. 9. Time evolution of some charge and hydrogenation states of circumovalene in the NCC 7023 NW PDR, at depths corresponding to $A_{V}=0$ a) and $A_{V}=1$ b), i.e. $\sim 42$ and $\sim 50^{\prime \prime}$ from the star, respectively. The typical timescale to reach steady state for charge and hydrogenation states are indicated with black arrows.

\subsection{Sensitivity to the astrophysical conditions}

Deriving the general trends of PAH evolution as a function of the astrophysical conditions from the case study of NGC 7023-NW is not straightforward, because the evolution of the physical conditions are correlated along the Star-NW cut. Therefore, in addition to the case study of NGC 7023, we also followed the method proposed by Le Page et al. (2001) and computed the charge and hydrogenated states of the four studied PAHs on a grid of astrophysical conditions. We used the interstellar radiation field (ISRF) of Mathis et al. (1983) as UV-visible spectrum, and normalised it to integrated intensities of $10^{n}(n=0,1,2,3,4)$ in Habing units. We considered a gas temperature of $100 \mathrm{~K}$, and several values of $n_{\mathrm{H}}$ between 10 and $10^{5} \mathrm{~cm}^{-3}$, with constant fractions of atomic hydrogen $n(\mathrm{H})=0.5 n_{\mathrm{H}}$ and free electrons $n\left(\mathrm{e}^{-}\right)=1.4 \times 10^{-4} n_{\mathrm{H}}$.

The results are presented in Fig. 10. They show that the evolution of the hydrogenation state of $\mathrm{C}_{54} \mathrm{H}_{18}$ and $\mathrm{C}_{66} \mathrm{H}_{20}$ exhibits a complex behaviour with $G_{0}$ and $n_{\mathrm{H}}$. On average, the slope of the boundary between the hydrogenated and dehydrogenated domains (solid line in Fig. 10) is clearly greater that $1(\sim 1.5$ and $\sim 2$ for circumcoronene and circumovalene, respectively) in a log$\log$ diagram. This results from the fact that, on average, roughly two photons are needed for $\mathrm{C}_{54} \mathrm{H}_{18}$ and $\mathrm{C}_{66} \mathrm{H}_{20}$ to reach their threshold energies for fragmentation at $\sim 17$ and $\sim 20 \mathrm{eV}$, respectively. When the intensity of the UV radiation field increases, the proportion of multiple absorption events increases and the probability of low energy photons being involved increases, which leads to slope changes with $G_{0}$ in Fig. 10.

Coronene is the only species for which we find that the hydrogenation state scales with $G_{0} / n_{\mathrm{H}}$, similar to the results reported by Le Page et al. (2003), who did not include multiphoton events in their model. This is because coronene has a low threshold energy for fragmentation $\left(E_{\text {th }} \approx 9.6<13.6 \mathrm{eV}\right)$ and therefore can easily dissociate after the absorption of a single photon. In other words, $G_{0} / n_{\mathrm{H}}$ is appropriate for quantifying the hydrogenation state of PAHs, but only in cases where dissociation is achieved with a single UV photon. However, in such cases dehydrogenation is so fast that it leads to the complete dehydrogenated product. Therefore, the population of interstellar PAHs must be dominated by species for which multi-photon events dominate the photodissociation process, and whose hydrogenation state is not well parametrised by $G_{0} / n_{\mathrm{H}}$.

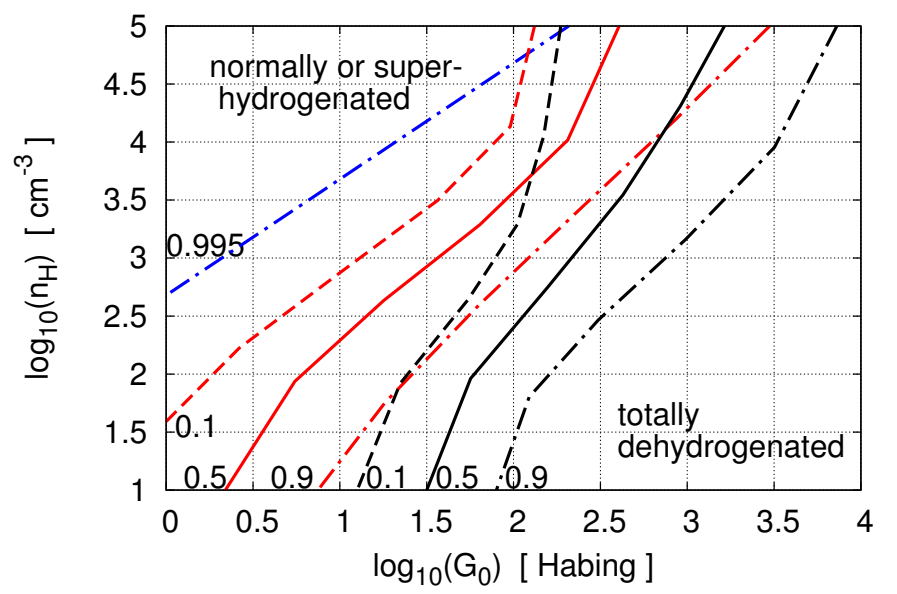

Fig. 10. Hydrogenation state of circumcoronene $\left(\mathrm{C}_{54} \mathrm{H}_{18}\right.$, red) and circumovalene $\left(\mathrm{C}_{66} \mathrm{H}_{20}\right.$, black) as a function of the intensity of the UV radiation field $G_{0}$ in Habing units and the local density $n_{\mathrm{H}}$. The lines labelled with 0.1 (respectively $0.5,0.9$ ) show the points for which $10 \%$ (respectively $50 \%, 90 \%$ ) of the species are totally dehydrogenated. For coronene, only the blue line labelled with 0.995 where $99.5 \%$ of coronene is fully dehydrogenated is represented, which is the dominant state of coronene for all the conditions spanned here.

\section{Discussion and perspectives}

\subsection{Consequences for the identification of a specific PAH in the ISM}

The detection of specific PAHs in the ISM has remained unsuccessful: Pilleri et al. (2009) reported an upper limit for rotational lines of corannulene $\mathrm{C}_{20} \mathrm{H}_{10}$ in the Red Rectangle (RR) nebula, and Kokkin et al. (2008) also report an upper limit on the abundance for hexa-peri-hexabenzocoronene $\mathrm{C}_{42} \mathrm{H}_{18}$ in the diffuse ISM from its optical emission. As a comparison, the results presented in Fig. 10 show that the larger, more compact, and therefore more photostable circumcoronene molecule $\mathrm{C}_{54} \mathrm{H}_{18}$ is expected to be at the edge between normally hydrogenated and fully dehydrogenated in physical conditions typical of the diffuse ISM (e.g. $G_{0} \sim 1$ and $n_{\mathrm{H}} \sim 10-100 \mathrm{~cm}^{-3}$ ). Thus it is very likely that $\mathrm{C}_{42} \mathrm{H}_{18}$ is completely dehydrogenated in the diffuse 
ISM, and this could explain why it was not detected by Kokkin et al. (2008).

In the particular case of $\mathrm{C}_{20} \mathrm{H}_{10}$ in the RR nebula, the situation is less clear since the radiation field of this object is remarkably poor in UV photons, so that Fig. 10 cannot be used for this object. Using the radiation field spectrum proposed by Mulas et al. (2006), a density of $n_{\mathrm{H}}=10^{5} \mathrm{~cm}^{-3}$ with an arbitrary fraction of molecular hydrogen of 0.5 , and a temperature of $100 \mathrm{~K}$ (Bujarrabal et al. 2005), our model predicts that coronene $\mathrm{C}_{24} \mathrm{H}_{12}$ is completely dehydrogenated in RR. It is therefore very likely that corannulene $\mathrm{C}_{20} \mathrm{H}_{10}$ cannot survive in $\mathrm{RR}$ as well. As a consequence, our results strongly suggest that future search for PAH spectroscopic signatures should focus on much larger species, containing at least 50 or 60 carbon atoms.

\subsection{The carbon content in PAHs and carbon clusters}

We predict a large abundance of carbon clusters in the cavity and in a significant fraction of the PDR of NGC 7023 NW. It raises the question of the evolution of carbon content in PAHs and carbon clusters. Carbon clusters have properties that differ significantly from those of PAHs, in terms of structure, stability, and spectroscopy. Therefore, when a PAH is photodissociated until full dehydrogenation, it can be considered as a loss of carbon content in the PAH population and a gain for the carbon cluster population. However, the latter population also may undergo evolution via photodissociation and carbon accretion. To get a first estimate, we used our model for computing the effective photodissociation rates (number of dissociation events per time unit) of planar $\mathrm{C}_{24}^{0 /+}, \mathrm{C}_{66}^{0 /+}$, and $\mathrm{C}_{96}^{0 /+}$ in the cavity and the NW PDR. We used the IR photon emission rates presented in Sect. 3.3.1 and computed the dissociation rates for the losses of $\mathrm{C}, \mathrm{C}_{2}$, and $\mathrm{C}_{3}$ using Eq. (3) and the parameters proposed by Léger et al. (1989) that are based on thermodynamic properties of graphite.

We found that the largest cluster in this study, $\mathrm{C}_{96}^{0 /+}$, is destroyed within a time longer than the age of the star, even at $10^{\prime \prime}$ from the star. $\mathrm{C}_{66}^{0 /+}$ needs more than several $10^{6}$ years to be significantly photodissociated in the NW PDR, and its destruction rate rises steeply in the cavity until very fast photodestruction $\left(\sim 10^{6} \mathrm{~s}^{-1}\right)$ because multiphoton events become frequent for the corresponding radiation field. $\mathrm{C}_{24}^{0 /+}$ photodissociates much faster than larger species in the NW PDR. Its photodissociation rate increases less rapidly than for larger species when going closer to the star, and reaches the same value as for $\mathrm{C}_{66}^{0 /+}$ close to the star. Here again, the evolution of the photodestruction rate is highly non-linear owing to the predominance of multiphoton events (threshold energies for fragmentation are $\sim 15,37,54 \mathrm{eV}$ for $\mathrm{C}_{24}^{0 /+}, \mathrm{C}_{66}^{0 /+}$, and $\mathrm{C}_{96}^{0 /+}$, respectively).

These results draw a picture in which the cavity is populated by large carbon clusters, while the PDR hosts a blend of large PAHs and relatively small carbon clusters. Carbon clusters are expected to explore various isomeric forms including non-planar (cage) ones (see for instance Jones \& Seifert 1997, for the various forms of $\mathrm{C}_{24}$ ). Heating by $\mathrm{UV}$ photons favours the exploration of the various isomeric forms. Our results are therefore consistent with the formation of the cage fullerene $\mathrm{C}_{60}$ in the cavity following the destruction of PAHs, as suggested by Berné $\&$ Tielens (2012).

In addition, this raises the question of the contribution of carbon clusters to the AIBs, as well as to the physical evolution of PDRs, e.g. through the photoelectric effect. Further consideration of these questions calls for more laboratory studies on the photophysical and chemical properties of these species.

\subsection{Perspectives}

In this paper, we have focussed on the interaction with photons and the reactivity with electrons and hydrogen. Including a larger set of chemical processes would be of interest for future studies.

The reactivity of PAHs with $\mathrm{C}^{+}$has been studied experimentally by Canosa et al. (1995). The authors found the reaction to proceed at a rate close to the rate of Langevin with, in the case of anthracene, two possible channels of comparable branching ratio: charge exchange (c.e.) and carbon accretion. In Sect. 5.3, we have evaluated the timescale for c.e. to be around 8 years in the conditions of NGC $7023 \mathrm{NW}$. A similar timescale is expected for carbon accretion. These values are shorter than the timescale for reaching hydrogenation steady state, which indicates that the corresponding reactions have to be included in a PAH chemical model. Still, reactions of PAHs with $\mathrm{C}^{+}$have only been studied for small PAHs and significant variations with the size of PAHs are observed in the branching ratio between c.e. and carbon accretion, calling for investigation of the reactivity of larger PAHs, including dehydrogenated PAHs, with $\mathrm{C}^{+}$.

PAHs can also react with atomic oxygen but these reactions are also poorly known. Le Page et al. (1999a) considered a reaction rate for PAH cations of $k_{+} \sim 10^{-10} \mathrm{~cm}^{3} \mathrm{~s}^{-1}$ for the addition of an $\mathrm{O}$-atom, regardless of the size of $\mathrm{PAH}$ cations. Assuming a density $n_{\mathrm{H}}=10^{4} \mathrm{~cm}^{-3}$ and an $\mathrm{O} / \mathrm{H}$ ratio of $3 \times 10^{-4}$, this leads to a reaction timescale of $\sim 100$ years. Therefore, for large PAHs, reaction with oxygen could play a significant role in PAH evolution.

Finally, it might be interesting to investigate the possibility that PAHs form clusters and/or complexes with heavy atoms, such as iron and silicon as proposed by several authors (Rapacioli et al. 2006; Simon \& Joblin 2009; Joalland et al. 2009)

\section{Conclusion}

In this paper, we have presented a new model dedicated to the evolution of the hydrogenation and charge states of PAHs. This model was designed to compute the time evolution of the internal energy of species, so that multi-photon dissociations, in the sense of the successive absorptions of several photons before the species could completely cool down, are taken into account for every dissociation process. We applied this model to four compact PAHs, namely coronene $\left(\mathrm{C}_{24} \mathrm{H}_{12}\right)$, circumcoronene $\left(\mathrm{C}_{54} \mathrm{H}_{18}\right)$, circumovalene $\left(\mathrm{C}_{66} \mathrm{H}_{20}\right)$ and circumcircumcoronene $\left(\mathrm{C}_{96} \mathrm{H}_{24}\right)$, in the conditions of NGC 7023 , both in the NW PDR and the cavity, and of the diffuse ISM.

The calculated spatial evolution of the PAH charge state is in good agreement with the observational constraints. The hydrogenation state is more difficult to predict as it depends on a wealth of processes, many of which have not or poorly been studied in the laboratory or theoretically. Clear observational diagnostics of the hydrogenation state of PAHs are also missing. Despite these difficulties, we showed that the general behaviour of PAH evolution is quite robust with molecular size and with the assumptions regarding the molecular processes. It leads to four major populations: superhydrogenated PAHs, normally hydrogenated PAHs, partially dehydrogenated PAHs and carbon clusters (i.e. totally dehydrogenated PAHs).

Partially dehydrogenated PAHs are found to be minor species in terms of abundance regardless of the molecular size 
or the position in the PDR. Superhydrogenated PAHs dominate only for very large PAHs $\left(N_{\mathrm{C}} \gtrsim 96\right)$ in the conditions of the NGC 7023 NW PDR. The size limit below which normally hydrogenated PAHs fully dehydrogenate to give birth to carbon clusters is found to lie between $N_{\mathrm{C}} \sim 50$ and 70 at the emission peak of AIBs, depending on the reaction rates of PAHs with $\mathrm{H}$ and $\mathrm{H}_{2}$. In the more diffuse medium of the cavity, where the fullerene $\mathrm{C}_{60}$ has recently been observed, we predict that all four species studied here are fully dehydrogenated.

We evaluated that the electronic recombination of $\mathrm{PAH}$ cations with electrons and the reactivity of PAHs with $\mathrm{H}_{2}$ are the main contributors to the uncertainties on our results. In addition, we were able to rationalise the non detection of corannulene $\mathrm{C}_{20} \mathrm{H}_{10}$ in the Red Rectangle nebula and of hexaperi-hexabenzocoronene $\mathrm{C}_{42} \mathrm{H}_{18}$ in the diffuse ISM. Therefore future searches to identify individual PAHs should focus on larger species that bear at least 50-60 carbon atoms.

More generally, multi-photon events were found to dominate the evolution of interstellar PAHs, even for the relatively low radiation fields that prevail in the diffuse ISM. This results in complex behaviour with variations in $n_{\mathrm{H}}$ and $G_{0}$, which cannot be simply parametrised with the ratio $G_{0} / n_{\mathrm{H}}$.

We also determined PAH evolution timescales to be much longer than previously thought. In some cases, timescales comparable to dynamical evolution timescales are reached, raising the question of the coupling between the dynamics and chemical evolution of PAHs. This could affect both the physics and chemistry of PDRs, for example through photoelectric heating and $\mathrm{H}_{2}$ formation on PAHs. Another important consequence of these long timescales is that reactions with species like $\mathrm{O}$ and $\mathrm{C}^{+}$ may occur on similar or shorter timescales and therefore require more investigation.

Finally, we showed that a large population of carbon clusters could exist in PDRs as a result of the dehydrogenation of PAHs. Our results therefore call for fundamental studies of carbon clusters to determine to what extent these species contribute to the mid-IR emission and affect the physical and chemical evolution of PDRs. These studies are also necessary to unveil the link between PAHs and fullerenes.

Acknowledgements. J. Montillaud acknowledges the support of the French Agence Nationale de la Recherche (ANR), under grant GASPARIM "Gas-phase PAH research for the interstellar medium", as well as the support of the Academy of Finland grant No. 250741. This work was supported by the French National Programme Physique et Chimie du Milieu Interstellaire, which is gratefully acknowledged. Some of the data presented in this paper were obtained from the Multimission Archive at the Space Telescope Science Institute (MAST). STScI is operated by the Association of Universities for Research in Astronomy, Inc., under NASA contract NAS5-26555. Support for MAST for non-HST data is provided by the NASA Office of Space Science via grant NAG5-7584 and by other grants and contracts.

\section{Appendix A: Modelling the internal energy of PAHs}

In this work, we model the time evolution of both the abundances and internal energies of the studied species. The latter is described as a histogram in which each bin $i$ represents the number per unit of volume of species bearing an internal energy between $E_{i-1}=i \Delta E$ and $E_{i}=(i+1) \Delta E$, where $\Delta E$ was set to $0.25 \mathrm{eV}$. In this appendix, we explain how we connect the rates characterising the fluxes of species from one bin to another, which are driven by UV-visible photon absorption, IR photon emission, and molecule dissociation.

\section{A.1. Heating molecules}

Species are heated after the non-ionizing absorption of a UV-visible photon. This leads to a transition from one bin $i$ to a higher bin $i^{\prime}\left(i^{\prime}>i\right)$, at a rate that depends on the flux of UV-visible photons $\mathcal{F}(h v)$, the absorption cross-section of the species $\sigma_{\text {abs }}(h v)$, and the non-ionization yield $1-Y_{\text {ion }}(h v)$. It also depends on the triangle-shaped probability function $\mathcal{P}_{i, i^{\prime}}(h v)$. This function expresses the probability that the energy $h v$ of the photon falls between the minimum $\left(E_{i^{\prime}-1}-E_{i}\right)$ and maximum $\left(E_{i^{\prime}}-E_{i-1}\right)$ differences between the boundaries of bins $i$ and $i^{\prime}$. Therefore, the rate of transitions from bin $i$ to bin $i^{\prime}$ is given by

$k_{\mathrm{abs}}^{i, i^{\prime}}=\int_{E_{i^{\prime}-1}-E_{i}}^{E_{i^{\prime}}-E_{i-1}} \mathcal{P}_{i, i^{\prime}}(h v)\left(1-Y_{\text {ion }}(h v)\right) \sigma_{\mathrm{abs}}(h v) \mathcal{F}(h v) \mathrm{d}(h v)$.

This expression is accurate, as long as one can neglect the variations in $\sigma_{\text {abs }}$ and $Y_{\text {ion }}$ with the internal energy of the species and the variations in abundance within the bin $i$. The latter condition requires $\Delta E$ to be smaller than $h v$ for the less energetic absorbed photons that have a significant impact on the internal energy of the species. This is a much weaker constraint than the conditions for a proper description of the cooling of molecules.

\section{A.2. Cooling molecules}

Species cool down through the emission of IR photons. This corresponds to the transition from one bin $i^{\prime}$ to a lower bin $i\left(i^{\prime}>i\right)$, at a rate that depends both on the IR emission rate and on the energy carried by the emitted photon that has to fall between the minimum and maximum differences between the boundaries of bins $i$ and $i^{\prime}$. We define the factor $\delta_{i, i^{\prime}}(E)$ that equals 1 when the latter condition is true, and 0 otherwise. For the sake of simplicity, and to ensure that the computations are tractable, we neglect the dispersion of the IR energies of the emitted photons and compute $\delta_{i, i^{\prime}}(E)$ using the mean IR energy as presented in Sect. 3.3.1. Therefore, the effective cooling rate from bin $i^{\prime}$ to bin $i$ is given by

$k_{\mathrm{IR}}^{i^{\prime}, i}=\int_{E_{i^{\prime}-1}}^{E_{i^{\prime}}} \delta_{i, i^{\prime}}(E) k_{\mathrm{IR}}(E) \mathrm{d} E$.

This formulation requires using thinner bins than the less energetic IR photon to ensure that each emission leads to an actual cooling.

\section{A.3. Molecule dissociation}

The dissociation rate from the bin $i$ of a species is computed as the mean value of the dissociation rate of the species over the width $\Delta E$ of the bin

$k_{\mathrm{diss}}^{i}=\int_{i \Delta E}^{(i+1) \Delta E} k_{\mathrm{diss}}(E) \mathrm{d} E / \Delta E$.

This approximate expression is better for lower values of $\Delta E$.

\section{A.4. Width of energy bins}

We empirically found that $\Delta E=0.25 \mathrm{eV}$ provides abundances accurate at $\sim 5 \%$ of the asymptotic results obtained when $\Delta E \rightarrow$ 0 . This value is therefore a good compromise between accuracy and computing time. 


\section{References}

Abouelaziz, H., Gomet, J. C., Pasquerault, D., Rowe, B. R., \& Mitchell, J. B. A. 1993, J. Chem. Phys., 99, 237

Alecian, E., Catala, C., Wade, G. A., et al. 2008, MNRAS, 385, 391

Allain, T., Leach, S., \& Sedlmayr, E. 1996a, A\&A, 305, 602

Allain, T., Leach, S., \& Sedlmayr, E. 1996b, A\&A, 305, 616

Bakes, E. L. O., \& Tielens, A. G. G. M. 1994, ApJ, 427, 822

Banisaukas, J., Szczepanski, J., Eyler, J., \& Vala, M. 2004, J. Phys. Chem. A, 108,3723

Barker, J. R. 1983, Chem. Phys., 77, 301

Berné, O., \& Tielens, A. G. G. M. 2012, PNAS, 109, 401

Berné, O., Joblin, C., Deville, Y., et al. 2007, A\&A, 469, 575

Berné, O., Joblin, C., Rapacioli, M., et al. 2008, A\&A, 479, L41

Betts, N. B., Stepanovic, M., Snow, T. P., \& Bierbaum, V. M. 2006, AJ, 651, L129

Biennier, L., Alsayed-Ali, M., Foutel-Richard, A., et al. 2006, Faraday Discussions, 133, 289

Bierbaum, V. M., Le Page, V., \& Snow, T. P. 2011, in PAHs and the Universe, eds. C. Joblin, \& A. G. G. M. Tielens, EAS Pub. Ser., 46, 427

Boissel, P., de Parseval, P., Marty, P., \& Lefèvre, G. 1997, J. Chem. Phys., 106, 4973

Bujarrabal, V., Castro-Carrizo, A., Alcolea, J., \& Neri, R. 2005, A\&A, 441, 1031 Canosa, A., Laube, S., Rebrion, C., et al. 1995, Chem. Phys. Lett., 245, 407

Castelli, F., \& Kurucz, R. L. 2004, New Grids of ATLAS9 Model Atmospheres

Dibben, M., Kage, D., Szczepanski, J., Eyler, J., \& Vala, M. 2001, J. Phys. Chem. A, 105,6024

Ekern, S., Marshall, A., Szczepanski, J., \& Vala, M. 1998, J. Phys. Chem. A, 102,3498

Fitzpatrick, E. L., \& Massa, D. 2005, AJ, 130, 1127

Fuente, A., Martin-Pintado, J., Neri, R., Rogers, C., \& Moriarty-Schieven, G. 1996, A\&A, 310, 286

Fuente, A., Martín-Pintado, J., Rodríguez-Fernández, N. J., et al. 1999, AJ, 518, L45

Gerin, M., Phillips, T. G., Keene, J., Betz, A. L., \& Boreiko, R. T. 1998, AJ, 500, 329

Goicoechea, J. R., Joblin, C., Contursi, A., et al. 2011, A\&A, 530, L16

Habart, E., Boulanger, F., Verstraete, L., et al. 2003, A\&A, 397, 623

Habart, E., Abergel, A., Boulanger, F., et al. 2011, A\&A, 527, 122

Habing, H. J. 1968, Bull. Astron. Inst. of the Netherlands, 19, 421

Hassouna, M., Le Garrec, J., Rebrion-Rowe, C., Travers, D., \& Rowe, B. 2003

Reactions of electrons with hydrocarbon cations: From linear alkanes to aromatic species, ed. S. Guberman (New York: Kluwer Academic/Plenum Publ) Herbst, E. 2001, Chem. Soc. Rev., 30, 168

Herbst, E., \& McEwan, M. J. 1990, A\&A, 229, 201

Ho, Y., Dunbar, R., \& Lifshitz, C. 1995, J. Am. Chem. Soc., 117, 6504

Hollenbach, D. J., \& Tielens, A. G. G. M. 1999, Rev. Mod. Phys., 71, 173

Joalland, B., Simon, A., Marsden, C. J., \& Joblin, C. 2009, A\&A, 494, 969

Joblin, C., \& Tielens, A. G. G. M. 2011, PAHs and the Universe, EAS Pub. Ser. edn., 46 (Toulouse)

Joblin, C., Léger, A., \& Martin, P. 1992, AJ, 393, L79

Joblin, C., Toublanc, D., Boissel, P., \& Tielens, A. G. M. 2002, Mol. Phys., 100, 3595

Joblin, C., Pilleri, P., Montillaud, J., et al. 2010, A\&A, 521, L25
Jochims, H. W., Ruhl, E., Baumgartel, H., Tobita, S., \& Leach, S. 1994, ApJ, 420, 307

Jones, R. O., \& Seifert, G. 1997, Phys. Rev. Lett., 79, 443

Kokkin, D. L., Troy, T. P., Nakajima, M., et al. 2008, ApJ, 681, L49

Le Bourlot, J., Le Petit, F., Pinto, C., Roueff, E., \& Roy, F. 2012, A\&A, 541, 76

Le Page, V., Keheyan, Y., Snow, T. P., \& Bierbaum, V. M. 1999a, Int. J. Mass Spectrom., 185, 949

Le Page, V., Keheyan, Y., Snow, T. P., \& Bierbaum, V. M. 1999b, J. Am. Chem. Soc., 121, 9435

Le Page, V., Snow, T. P., \& Bierbaum, V. M. 2001, ApJSS, 132, 233

Le Page, V., Snow, T. P., \& Bierbaum, V. M. 2003, AJ, 584, 316

Le Petit, F., Nehmé, C., Le Bourlot, J., \& Roueff, E. 2006, ApJSS, 164, 506

Léger, A., D’Hendecourt, L., Boissel, P., \& Désert, F. X. 1989, A\&A, 213, 351

Ling, Y., \& Lifshitz, C. 1998, J. Phys. Chem. A, 102, 708

Ling, Y., Gotkis, Y., \& Lifshitz, C. 1995, Eur. J. Mass Spectrom., 1, 41

Malloci, G., Mulas, G., \& Joblin, C. 2004, A\&A, 426, 105

Malloci, G., Joblin, C., \& Mulas, G. 2007, A\&A, 462, 627

Malloci, G., Mulas, G., Cecchi-Pestellini, C., \& Joblin, C. 2008, A\&A, 489, 1183

Mathis, J. S., Mezger, P. G., \& Panagia, N. 1983, A\&A, 128, 212

Mebel, A. M., Lin, M. C., Yu, T., \& Morokuma, K. 1997, J. Phys. Chem. A, 101, 3189

Mermilliod, J., Mermilliod, M., \& Hauck, B. 1997, A\&ASS, 124, 349

Mulas, G., Malloci, G., Joblin, C., \& Toublanc, D. 2006, A\&A, 460, 93

Novotny, O., Sivaraman, B., Rebrion-Rowe, C., et al. 2005, J. Chem. Phys., 123, 104303

Okada, Y., Pilleri, P., Berné, O., et al. 2013, A\&A, accepted

Petrie, S., Javahery, G., \& Bohme, D. K. 1992, J. Am. Chem. Soc., 114, 9205

Pilleri, P., Herberth, D., Giesen, T. F., et al. 2009, MNRAS, 397, 1053

Pilleri, P., Montillaud, J., Berné, O., \& Joblin, C. 2012, A\&A, 542, A69

Rapacioli, M., Joblin, C., \& Boissel, P. 2005, A\&A, 429, 193

Rapacioli, M., Calvo, F., Joblin, C., et al. 2006, A\&A, 460, 519

Rebrion-Rowe, C., Le Garrec, J., Hassouna, M., Travers, D., \& Rowe, B. 2003, Int. J. Mass Spectrom., 223, 237

Rogers, C., Heyer, M. H., \& Dewdney, P. E. 1995, ApJ, 442, 694

Ruiterkamp, R., Halasinski, T., Salama, F., et al. 2002, A\&A, 390, 1153

Ruiterkamp, R., Cox, N. L. J., Spaans, M., et al. 2005, A\&A, 432, 515

Salama, F., Galazutdinov, G. A., Kreowski, J., et al. 2011, ApJ, 728, 154

Sellgren, K., Werner, M. W., Ingalls, J. G., et al. 2010, ApJ, 722, L54

Simon, A., \& Joblin, C. 2009, J. Phys. Chem. A, 113, 4878

Skrutskie, M. F., Cutri, R. M., Stiening, R., et al. 2006, AJ, 131, 1163

Snow, T. P., Le Page, V., Keheyan, Y., \& Bierbaum, V. M. 1998, Nature, 391, 259

Spitzer, L. 1978, Physical processes in the interstellar medium

Stein, S. E., \& Rabinovitch, B. S. 1973, J. Chem. Phys., 58, 2438

Szczepanski, J., Dibben, M., Pearson, W., Eyler, J., \& Vala, M. 2001, J. Phys. Chem. A, 105, 9388

Tielens, A. G. G. M. 2005, The Physics and Chemistry of the Interstellar Medium Verstraete, L., Léger, A., D’Hendecourt, L., Defourneau, D., \& Dutuit, O. 1990, A\&A, 237, 436

Visser, R., Geers, V. C., Dullemond, C. P., et al. 2007, A\&A, 466, 229

Wolfire, M. G., Tielens, A. G. G. M., Hollenbach, D., \& Kaufman, M. J. 2008, ApJ, 680, 384 